\title{
Physics of Coevolution of Galaxies and Supermassive Black Holes
}

\author{
Renyue Cen $^{1}$
}

\begin{abstract}
A new model for coevolution of galaxies and supermassive black holes (SMBH) is presented that is physically based. The evolutionary track starts with an event that triggers a significant starburst in the central region of a galaxy. In this model, the main SMBH growth takes place in post-starburst phase fueled by recycled gas from inner bulge stars in a self-regulated fashion on a time scale that is substantially longer than 100 Myrs and at a diminishing Eddington ratio with time. We argue that the SMBH cannot gorge itself during the starburst phase, despite the abundant supply of cold gas, because star formation is a preferred mode of gas consumption in such an environment than accretion to the central SMBH. We also show that feedback from star formation is at least as strong as that from AGN and thus, if star formation is in need of being quenched, AGN feedback generally does not play the primary role. The predicted relation between SMBH mass and bulge mass/velocity dispersion is consistent with observations. A clear prediction is that early-type galaxy hosts of high Eddingtion rate AGNs are expected to be light-blue to green in optical color, gradually evolving to the red sequences with decreasing AGN luminosity. A suite of falsifiable predictions and implications with respect to relationships between various types of galaxies and AGN, and others, are made. For those where comparisons to extant observations are possible, the model appears to be in good standing.
\end{abstract}

Subject headings: Methods: analytic, ISM: kinematics and dynamics, Galaxies: interactions, Galaxies: evolution, Supermassive black holes

\section{Introduction}

The tight correlation between galactic center supermassive black hole (SMBH) mass $\left(\mathrm{M}_{\mathrm{BH}}\right)$ and the bulge mass $\left(\mathrm{M}_{\mathrm{BG}}\right)$ or velocity dispersion $(\sigma)$ in the nearby universe (e.g., Richstone et al. 1998; Ferrarese \& Merritt 2000; Tremaine et al. 2002) strongly suggests coevolution of the two classes, at least over the Hubble time. In many semi-analytic calculations one of the most adopted assumptions, to put it simply, is that active galactic nuclei

\footnotetext{
${ }^{1}$ Princeton University Observatory, Princeton, NJ 08544; cen@astro.princeton.edu
} 
(AGN) feedback is able to prevent most of the gas from accreting onto the SMBHs and at the same time is able to fix most of the "defects" of galaxy formation models such as the shape of the galaxy luminosity function and star formation (SF) history (e.g., Kauffmann \& Haehnelt 2000; Croton et al. 2006; Somerville et al. 2008) with the underlying feedback physics parameterized. The substantial success in explaining a variety of observations enjoyed by these semi-analytic models is indicative of the relevance of AGN feedback. Calculations of the coupled evolution of SMBHs and galaxies using three-dimensional hydrodynamic simulations deploy thermal energy feedback in regions significantly outside of the Bondi radius of the putative SMBH that effectively couples to the surroundings to regulate the SF and eventually drive the gas away. These pioneering detailed simulations have provided much physical insight and appear to be remarkably successful in accounting for many intricate observables, including AGN light curves, Eddington ratio distributions and SMBH-bulge relation and its scatter, for certain chosen value of the feedback energy strength (e.g., Di Matteo et al. 2005; Hopkins et al. 2006). What is hitherto left open in these calculations is the physical origin of the adopted energy feedback. One concern is that the derived SMBHbulge relation depends very sensitively on the adopted energy feedback parameter due to the strong radiative cooling (e.g., Silk \& Nusser 2010; Choi \& Ostriker 2011). Thus, it is prudent to seek underlying physical origins for these successful models and, before that is achieved, continue to explore alternative models.

This paper synthesizes an alternative physical model largely based on known physics. Before describing our overall model, we shall first, in $\S 2$, examine the plausibility of the fundamental claim that AGN feedback is primarily responsible for regulating not only $\mathrm{SMBH}$ growth but also SF. We argue that scenarios invoking AGN as the primary "blowing machine" during the intense starburst phase may logically require significant fine-tuning. We then describe the evolutionary path from a starburst to an elliptical galaxy, including the coupled evolution of star formation and SMBH growth in the ensuing two sections.

In $\S 3$, we show that growth of SMBH during the starburst phase is limited and constitutes a small fraction of the overall SMBH consumption. The physical reason is that this phase is over-supplied with gas such that only a very small central disc is gravitationally stable (Toomre parameter $Q>1$ ) for gas accretion onto the $\mathrm{SMBH}$, while all other regions are unstable and more conducive to star formation. Since the SF time scale is much shorter than the Salpeter accretion time scale, most of the gas forms into stars. The accreted mass during this phase is probably limited to a few percent of the final SMBH mass. In $\S 4$, we point out that energy or momentum feedback from SF is at least as competitive as that from the AGN during the starburst phase. Therefore, SF is largely responsible for blowing most of the last patch of gas away to end the starburst phase. In short, during the starburst phase, the SMBH does not grow significantly and does not play the leading role in quenching the star formation. 
In $\S 5$, we show that most of the growth of the SMBH occurs in the ensuing poststarburst period, when the bulge/elliptical galaxy is largely in place and SF enters "passive" evolution. The fuel for this primary growth phase is provided by the gas recycled back into the interstellar medium (ISM) from aging bulge stars, proposed earlier by Norman \& Scoville (1988) in the context of a central stellar cluster and stressed recently by Ciotti \& Ostriker (2007) in the context of elliptical galaxies. It provides a relatively "diffuse" (compared to the starburst phase) but steady gas supply that, we show, is ideal for feeding SMBH via an accretion disc. Meanwhile, SF is the dominant mode for gas consumption in the outer region because the accretion is unstable to fragmentation there, even in this phase. Selfregulation is at work for the growth of the $\mathrm{SMBH}$ during this period and is provided by much more robust (compared to energy feedback) radiation pressure induced momentum. The amplitude and slope of the resultant SMBH-bulge relation with this self-regulation is consistent with observations.

In this model, the entire evolution from the onset of starburst, due to a gas-rich merger or some significant event that drives a large amount of gas into the central region within a short period of time, to becoming a quiescent elliptical galaxy (or a bulge of a future spiral galaxy) consists of three distinct periods, as summarized in $§ 5.1$ and in Figure 2; (1) "Starburst Period": merger of two gas-rich spiral galaxies or some other significant event induces a starburst that lasts about $10^{7}-10^{8}$ yrs. The $\mathrm{SMBH}$ grows modestly during this period. The feedback energy/momentum from the starburst, i.e., supernovae, drives the last patch of gas away and helps shut down star formation. (2) "SMBH Prime Period": several hundred million years after the end of the starburst, aging low-to-intermediate mass stars, now in the form of red giants and other post-main-sequence states, start to return a substantial fraction of their stellar mass to the ISM. The SMBH accretion is mostly supply limited in most of this period, except during the first several hundred million years or so, and lasts for order of gigayear. Because the rate of gas return from stars diminishes with time, the Eddington ratio of the SMBH decreases with time and the SMBH spends most of the time during this period at low the Eddington ratio $\left(\leq 10^{-3}\right)$. The SMBH growth is nearly synchronous with star formation from recycled gas during this period. The accompanying star formation rate is quite substantial, roughly $\sim(5-10)\left(M_{*} / 10^{11} \mathrm{M}_{\odot}\right)(t / 1 \mathrm{Gyr})^{-1.3} \mathrm{M}_{\odot} \mathrm{yr}^{-1}$, where $t$ is time in Gyr and $M_{*}$ is stellar mass of the elliptical galaxy formed during the starburst (at $\left.t=0\right)$. The duration of this phase depends sensitively on the lower cutoff mass of the initial mass function (IMF). (3) "Quiescent Elliptical Galaxy": several gigayears after the end of the starburst the elliptical galaxy is now truly red and dead - gas return rate is now negligible so both accretion to the central SMBH and residual star formation have ceased. It is possible, at least for an elliptical galaxy that is not too massive (i.e., $M_{\text {tot }} \leq 10^{12} \mathrm{M}_{\odot}$ ), that it may grow a disk. The feeding of the central SMBH in the bulge of spiral galaxy during this period is no longer by aging stars, rather by occasional objects (molecular clouds, stars, etc) that happen to be on some plunging orbits due to secular or random events. 
We present some predictions and implications of this model in $\S 6.2-6.9$, followed by conclusions in $\S 7$. Where comparisons can be made between the predictions of the model and observations, they appear to be in good agreement. Some additional predictions could provide further tests of the model.

\section{AGN Cannot Regulate Star Formation During Starburst}

While the subsequent sections of quantitative physical analysis are independent of statements made in this section, we shall argue for the assertion in the title of this section with logic, in hopes of being able to provide some conceptual clarity to the role of AGN feedback on star formation during the starburst phase. The starting point of the evolutionary sequence is a starburst. It may be triggered by a major merger of two gas-rich galaxies or by other significant events that channel a large amount of gas into the central region in a short period of time. Consider that an event causes a large amount of gas of mass $\mathrm{M}_{\text {gas }}$ to land in the central region. Physical processes then operate on the gas to produce a starburst, accompanied by some growth of the central SMBH, along with some associated feedback from both. Extreme events of this kind may be identified with observed Ultra-Luminous InfraRed Galaxies (ULIRGs) (e.g., Sanders et al. 1988) or Sub-Millimeter Galaxies (SMGs) (e.g., Chapman et al. 2005). Theoretical models (e.g, Silk \& Rees 1998; Hopkins et al. 2006) have proposed that feedback from AGN is responsible for the regulation of SF and SMBH growth so as to produce the observed Magorrian et al. (1998) relation where the ratio of the final SMBH to bulge stellar mass is $\mathrm{M}_{\mathrm{BH}}: \mathrm{M}_{\mathrm{BG}} \sim 2: 1000$. We shall now re-examine this case.

Consider how the infallen gas may be partitioned. Mass conservation requires $\mathrm{M}_{\mathrm{BH}}+$ $\mathrm{M}_{\mathrm{BG}}+\mathrm{M}_{\text {out }}=\mathrm{M}_{\text {gas }}$, where $\mathrm{M}_{\text {out }}$ is the amount of gas that is blown away from the bulge. Clearly, only a very small fraction of the initial gas $\mathrm{M}_{\text {gas }}$ can possibly end up in the central $\mathrm{SMBH}$, i.e., $f_{\mathrm{BH}} \equiv \mathrm{M}_{\mathrm{BH}} / \mathrm{M}_{\text {gas }} \ll 1$. Let us assume that the reason for a very small $f_{\mathrm{BH}}$ is because the feedback from the central SMBH prevented its own further growth during this phase. Since SMBH masses are observed to span a very wide range, it must be that this purported SMBH feedback process that regulates its own growth is galaxy specific, i.e., dependent on at least some physical variables characterizing the galaxy. A usual and reasonable assumption (which we are not advocating at the moment) for that is that either the gravitational potential well of the bulge or of the total halo determines the final SMBH mass, in coordination with its feedback.

Does SMBH feedback dominate that of starburst in terms of regulating both SMBH growth and starburst? While we will show later (in §3) that the answer is largely no to regulating the starburst at least, we assume that the answer is yes to both for the sake of continuing the present thought experiment. The simplified sequence of events then plays out 
more or less as follows. The central SMBH accretes gas and builds up its feedback strength until its mass has reached the observed value, then blows away all the remaining gas and both SMBH accretion and SF stop abruptly. What might have happened to SF during all this time before the gas is blown away? There are three possible scenarios. Scenario \#1, the SMBH accretion is so competitive and quick that most of the gas is blown away by the SMBH feedback before much SF has occured. That of course cannot have happened, because that would be inconsistent with the observed $\mathrm{M}_{\mathrm{BH}}-\mathrm{M}_{\mathrm{BG}}$ relation.

Scenario \#2, SF precedes at a pace that is in concert with the SMBH feedback such that by the time that $\mathrm{M}_{\mathrm{BH}}=0.002\left(\mathrm{M}_{\text {gas }}-\mathrm{M}_{\text {out }}\right)$, the amount of stars formed is equal to $\mathrm{M}_{\mathrm{BG}}=0.998\left(\mathrm{M}_{\text {gas }}-\mathrm{M}_{\text {out }}\right)$; the rest of gas of mass $\mathrm{M}_{\text {out }}$ got blown away by the feedback from the SMBH. This scenario is designed to match the observed $\mathrm{M}_{\mathrm{BH}}-\mathrm{M}_{\mathrm{BG}}$ relation. What remains undetermined is how large $f_{\text {out }} \equiv \mathrm{M}_{\text {out }} / \mathrm{M}_{\text {gas }}$ is. Is it close to 1 or 0 ? In the case $f_{\text {out }} \sim 1$, because $\left(1-f_{\text {out }}\right)$ is a small number, there is no particular preferred value for it. The potential well created by the eventual bulge stars would be much shallower than the original one already created by the residing gas. In other words, the SMBH only knew the potential well of the original gas and it would be rather arbitrary how much stars the SMBH decides to allow the bulge to have. If one argues that it is the potential well of the total halo mass that matters, the SMBH still did not know how to let SF take place at such a rate that we have the very tight observed $\mathrm{M}_{\mathrm{BH}}-\mathrm{M}_{\mathrm{BG}}$ relation for the bulge region. Thus, this case also appears to require much fine tuning. Besides, if $\left(1-f_{\text {out }}\right)$ is too small, the bulge will be too small compared to what is observed.

The opposite case with $f_{\text {out }} \ll 1$ is at least substantially more stable, since a large fraction of the original gas has formed into stars before the remainder of the gas got blown away. In this case the SMBH would "know better" the gravitational potential well eventually sustained by bulge stars, because it is not too far from that created by the initial gas. Then, how did the SMBH know when to blow away the remaining gas left over from SF and SMBH accretion? Should the SMBH blow away the gas when $f_{\text {out }}=0.90$ (an arbitrarily picked number for illustration purpose) or should it wait a bit longer to finally blow away the gas when $f_{\text {out }}=0.10$ ? It may require more energy or momentum in the former than the latter; but that can readily be accommodated by a proportionally increased amount of gas accreted, in the vein of feedback from SMBH providing the required feedback energy or momentum. Since the amount of gas available before $f_{\text {out }}=0.90$ is blown away in this hypothetical case is capable of growing the SMBH to be 900 more massive than observed and the amount of time available (cosmological scale) is much longer than Salpeter time, there is no obvious reason why the SMBH cannot grow 10 times (or whatever factor) larger to blow away the gas when $f_{\text {out }}=0.90$ instead of when $f_{\text {out }}=0.10$. How the SMBH has communicated with the bulge to ration the gas consumption would be a mystery. Thus, even in this case with $f_{\text {out }} \ll 1$, taking it as a given that the SMBH always stands ready to provide the necessary feedback, having $\mathrm{SMBH}$ feedback to regulate the overall SF in the bulge such that the ratio of the two matches 
the observation, again, requires a substantial amount of fine tuning. Nevertheless, since it is reasonable to expect that the dependence of the outcome, such as the $\mathrm{M}_{\mathrm{BH}}-\mathrm{M}_{\mathrm{BG}}$ relation, on any proposed feedback processes (including those based on thermal energy deposition near the galaxy center) is likely a monotonic function of the adopted feedback strength, it should be expected that a solution be found such that the observed $M_{\mathrm{BH}}-\mathrm{M}_{\mathrm{BG}}$ relation is obtained, for some chosen value of feedback strength, at least for some narrow range in $\mathrm{M}_{\mathrm{BG}}$. But, until there is clear physical reason or direct observational evidence to support the chosen value of the feedback parameter which the solution sensitively depends on, such an approach remains to be refined. We will provide an alternative, significantly less contrived, quantitative physical mechanism to circumvent this concern of fine tuning.

\section{Starburst Phase: Modest SMBH Growth and SF Shutdown by Stars}

We have argued in the previous section that AGN feedback cannot logically play the leading role in regulating $\mathrm{SF}$, in the sense that while some feedback from the SMBH can certainly affect its surrounding gas, there is no particular reason why this could provide a quite precise (within a factor of a few) rationing mechanism during the starburst phase so as to produce the observed relation between the two. We shall now argue for Scenario \#3: during the starburst phase the SF is self-regulated and self-limited, while SMBH growth is modest, does not need regulation and does not provide significant feedback to star formation.

We now give a physical reason for why, even though there is a very large supply of gas in the bulge region during the starburst phase, the SMBH growth is modest. We will make three simplifying assumptions to present trackable illustration without loss of generality. We assume (1) for the regions of interest a geometrically thin Keplerian disc dominated by the SMBH gravity (at least at the radii of interest here) is in a steady state, meaning the accretion rate (Frank et al. 1992):

$$
\dot{M}=3 \pi \nu \Sigma_{g}\left[1-\left(r_{\text {in }} / r\right)^{1 / 2}\right]^{-1} \approx 3 \pi \nu \Sigma_{g}
$$

is constant in radius $r$ and time, where $\Sigma_{g}$ is gas mass surface density and $\nu$ is viscocity; the last equality is valid because the radii of interest here are much larger than the radius of the inner disc $r_{\text {in }}$; note that it is inevitable to form a disk in the central given the rapid cooling and finite angular momentum; (2) we adopt the $\alpha$-disc viscosity (Shakura \& Sunyaev 1973):

$$
\nu=\alpha c_{s}^{2} \Omega^{-1}
$$

where $\alpha$ is a dimensionless viscosity constant for which magnetorotational instability process (Balbus \& Hawley 1991) provides a physical and magnitude-wise relevant value; $c_{s}$ is sound speed and $\Omega$ is angular velocity (equal to epicyclic frequency for Keplerian disc). The Toomre 
Q parameter of the gas disc can be obtained from Equations (1]2]:

$$
Q \equiv \frac{c_{s} \Omega}{\pi G \Sigma_{g}}=\frac{1}{3^{1 / 2} \pi^{3 / 2} \alpha^{1 / 2}}\left(\frac{\dot{M}}{\mathrm{M}_{\mathrm{BH}}}\right)^{1 / 2}\left(\frac{G^{-1 / 4} \mathrm{M}_{\mathrm{BH}}^{5 / 4}}{\Sigma_{g}^{3 / 2} r^{9 / 4}}\right)
$$

where $G$ is gravitational constant. The slope of the surface brightness profiles of the inner region of the observed powerlaw elliptical galaxies, which are assumed to be the product of the starbursts resulting from the gas-rich galaxy mergers, has a value concentrated in the range -1.0 to -0.5 (e.g., Faber et al. 1997; Kormendy et al. 2009), reproduced in merger simulations (e.g., Hopkins et al. 2009). Presumably the initial gas density profile is similar to the final observed stellar density profile in the inner regions. For ease of algebraic manipulations, we assume (3) the de Vaucouleur mass surface density profile (with a halfmass radius $r_{e}$ ) but with the inner region at $r \leq r_{p} \equiv 0.07 r_{e}$ modified to be a Mestel disc as:

$$
\Sigma_{g}(r)=\Sigma_{0}\left(\frac{r}{r_{0}}\right)^{-1} \quad \text { for } \quad r \leq r_{p}
$$

where $\Sigma_{0}$ is the normalizing surface density at some radius $r_{0}$; we will only be dealing with region $r \leq r_{p}$; the notional nuclear velocity dispersion of the system without the central $\mathrm{SMBH}$ at $r \leq r_{p}$ is related to $\Sigma_{0}$ and $r_{0}$ by

$$
\sigma_{n}^{2}=\pi G \Sigma_{0} r_{0}
$$

Subsequent results do not sensitively depend on the exact slope. The total mass of such a hybrid profile is equivalent to a truncated isothermal sphere with a truncation radius of $2 r_{e}$ and velocity dispersion on galactic scales of $\sigma_{g}$ such that

$$
\sigma_{n}=1.55 \sigma_{g}
$$

Since the dynamical time, say at $1 \mathrm{kpc}$ for a $200 \mathrm{~km} / \mathrm{s}$ bulge being only $5 \times 10^{6} \mathrm{yr}$, is much shorter than the Salpeter time, it is appropriate to assume that the gas disc is assembled instantaneously with respect to accretion to the SMBH when infalling gas lands on the disc. Combining Equations (34:|5|6) we rewrite $Q$ as

$$
Q=0.32 \alpha_{0.01}^{-1 / 2} \epsilon_{0.1}^{-1 / 2} l_{E}^{1 / 2} M_{8}^{5 / 4} \sigma_{200}^{-3} r_{\mathrm{pc}}^{-3 / 4}
$$

where $\alpha_{0.01}=\alpha / 0.01, \epsilon=0.1 \epsilon_{0.1}$ is the $\mathrm{SMBH}$ radiative efficiency, $l_{E}$ is Eddington ratio, $M_{8}=\mathrm{M}_{\mathrm{BH}} / 10^{8} \mathrm{M}_{\odot}, \sigma_{200}=\sigma_{g} / 200 \mathrm{~km} / \mathrm{s}, r_{\mathrm{pc}}=r / 1 \mathrm{pc}$. The value of $\alpha$ is quite uncertain, possibly ranging from $10^{-4}$ to 1 (e.g., Hawley et al. 1995; Brandenburg et al. 1995; Stone et al. 1996; Armitage 1998; Gammie 2001; Fleming \& Stone 2003; Fromang \& Papaloizou 2007). Setting $Q$ in Equation (7) to unity defines the disc stability radius

$$
r_{Q}=0.22 \alpha_{0.01}^{-2 / 3} \epsilon_{0.1}^{-2 / 3} l_{E}^{2 / 3} M_{8}^{5 / 3} \sigma_{200}^{-4} \mathrm{pc}
$$


within which $Q>1$ and disc is stable to gravitational fragmentation, and outside which $Q<1$ and disc is subject to gravitational fragmentation to form stars, supported by both simulations (e.g., Gammie 2001; Rice et al. 2003) and circumstantial observational evidence of the existence of stellar disc at small Galactic radius $(\sim 0.1 \mathrm{pc})$ (e.g., Levin \& Beloborodov 2003; Paumard et al. 2006). The demarcation value of $Q$ between stability and fragmentation does not appear to be qualitatively different even if the disc is under strong illumination (e.g., Johnson \& Gammie 2003), as might happen to a nuclear gas disc in the starburst phase. The disc mass within $r_{Q}$ is

$$
M_{Q}=9.8 \times 10^{6} \alpha_{0.01}^{-2 / 3} \epsilon_{0.1}^{-2 / 3} l_{E}^{2 / 3} M_{8}^{5 / 3} \sigma_{200}^{-2} \mathrm{M}_{\odot}
$$

This is the accretable mass out of the entire bulge region (note that some of the outer regions are more random motion supported). This conclusion reached is in good agreement with Goodman (2003), who employs somewhat different assumptions than in this study in that he assumes local energy balance, while we impose the observationally inferred inner density profile to be self-consistent; the good agreement suggests that this result is quite robust, insensitive to assumptions made. Taking cue from our own Galaxy, if we assume that the initial SMBH mass of the two merging spiral galaxies of mass $\sim 10^{12} \mathrm{M}_{\odot}$ each is $2.5 \times$ $10^{6} \mathrm{M}_{\odot}$, for a spiral galaxy of velocity dispersion of $200 \mathrm{~km} / \mathrm{s}$, we see that the amount of mass that could be readily accreted according to Equation (9) using $M_{8}=0.05$ is $6.7 \times$ $10^{4} \alpha_{0.01}^{-2 / 3} \epsilon_{0.1}^{-2 / 3} l_{E}^{2 / 3} \mathrm{M}_{\odot}$. Note that the final SMBH mass for such a system is $\sim 1.3 \times 10^{8} \mathrm{M}_{\odot}$ (Tremaine et al. 2002), if we were to match the observations.

It is possible that the mass accreted to the SMBH may be larger than that indicated by Equation (9) due to replenishment. Replenishment of low angular momentum gas during the starburst phase may be possible in two ways: (1) through orbital decay of outer disc gas or (2) direct infall of low-J gas from outer regions. We will show below that (1) does not significantly increase the accretable mass. Process (2) is probably unavoidable to some extent but unlikely to be frequent enough to be significant for the following reasons. All the low angular momentum infalling gas falls into the inner regions initially according to its respective specific angular momentum driven by the torque of the trigger event (e.g., merger or some other significant torquing event). To replenish low angular momentum gas directly to the central region some frequent and significant torquing events during the starburst phase are needed. It seems unlikely that such events will be frequent enough to be able to reach the observed final SMBH mass: about $\sim 100-1000$ replenishments will be required. One might approximately equate the number of replenishment (i.e., significant disturbance) to the number of generations of stars formed during the starburst phase (by assuming that each generation of star formation manages to redistribute the angular momentum of a significant fraction of the gas), which is unlikely to be close to $\sim 100-1000$. In summary, taking into account possible additional accretion due to some replenishment and giving the benefit of the possibility of $\alpha_{0.01}<1$, it seems improbable that the $\mathrm{SMBH}$ is able to acquire a mass during the starburst phase that would be much more than $10 \%$ of the final value. 
At $r \geq r_{Q}$, the disc is unstable to SF. For SF under the conditions relevant here both the dynamical and cooling time are short and do not constitute significant bottleneck; if they were the only time scale bottleneck, SF would be too efficient. A possible bottleneck for SF is the time scale to rid the cloud of the magnetic flux (assuming the SF clouds are initially magnetically sub-critical). The main ionization source in the depth of molecular cloud cores is cosmic rays $(\mathrm{CR})$. While the exact ionization rate by $\mathrm{CR}$ is unknown for other cosmic systems, we have some estimate of that for our own Galaxy, $\zeta_{\mathrm{CR}, \mathrm{Gal}}=(2.6 \pm 1.8) \times 10^{-17} \mathrm{~s}^{-1}$ (e.g., van der Tak \& van Dishoeck 2000). If one assumes that the CR ionization rate in starburst is 100 times (modeling a typical ULIRG in this case) that of the Galactic value, considering that the SF rate in ULIRGs is $100-1000$ times the Galactic value occuring in a more compact region and that the CR in ULIRGs may be advected out via fast galactic winds (versus slow diffusion in the Galaxy), one may roughly estimate that the ambipolar diffusion time is $7 \times 10^{6} \mathrm{yr}$ at a density of $n \sim 10^{5} \mathrm{~cm}^{-3}$ using standard formulas for recombinations (e.g., McKee \& Ostriker 2007). This estimate is, however, uncertain. We will again look to direct observations to have a better gauge. Gao \& Solomon (2004) show, from HCN observations, that ULIRGs and LIRGs convert molecular gas at $n \geq 3 \times 10^{4} \mathrm{~cm}^{-3}$ at an e-folding time scale of $t_{\mathrm{SF}} \sim 2 \mathrm{Myr}$, consistent with the above rough estimate. It is clear that SF time scale is much shorter than the Salpeter time of $4.5 \times 10^{7} \epsilon_{0.1} \mathrm{yr}$; in other words, when gas is dense and unstable, star formation competes favorably with the SMBH accretion with respect to gas consumption. Therefore, most of the gas at $r \geq r_{Q}$ will be depleted by SF. When the density profile of the disc at $r \geq r_{Q}$ steepens to be $\Sigma_{g}(r)=\Sigma_{Q}\left(r / r_{Q}\right)^{-5 / 2}$, where $\Sigma_{Q}$ is the gas surface density at $\sim r_{Q}$, the disc at $r \geq r_{Q}$ may become stable again. While continued accretion supplied by gas on the outer disc is likely, albeit at a much lower level, the mass integral is convergent and most of the mass of this outer disc is at $r_{Q}$ given the density slope, even if the entire outer disc at this time is accreted.

Thus, it appears that the amount of gas that is actually accreted by the SMBH during the starburst phase is rather limited. This new and perhaps somewhat counter-intuitive conclusion is strongly supported by available observations of ULIRGs. This conclusion is also opposite to most models that rely on SMBH to provide the necessary feedback to regulate star formation (e.g, Silk \& Rees 1998; Hopkins et al. 2006). Observational evidence is that the SMBHs in ULIRGs and SMGs appear to be significantly smaller (an order of magnitude or more) than what the $\mathrm{M}_{\mathrm{BH}}-\mathrm{M}_{\mathrm{BG}}$ relation would suggest (e.g., Genzel et al. 1998; Ivison et al. 2000; Ptak et al. 2003; Ivison et al. 2004; Alexander et al. 2005a. b; Kawakatu et al. 2006; Alexander et al. 2008). Nonetheless, it is expected that the AGN contribution in ULIRGs should become relatively more important for larger more luminous galaxies (see Equation 9), consistent with observations (e.g., Lutz et al. 1998). Starbursts occuring on rotating nuclear disc/rings in ULIRGs are also supported by circumstantial observational evidence (e.g., Downes \& Solomon 1998).

The overall conclusion that the SMBH feedback has little effect on the amount of stars 
formed is in agreement with that of DeBuhr et al. (2010) who investigated the radiation pressure-regulated SMBH feedback in the starburst phase of the merger simulations utilizing a sub-grid model for SMBH accretion. One specific common outcome between our calculation and their simulation is that most of the gas formed into stars, regardless of the feedback strength. A notable difference between our calculation and theirs is that their simulation resolution, a gravitational softening length of $47 \mathrm{pc}$, is significantly larger than $r_{Q}$ (Equation 8). As a result, it is possible that their simulations do not resolve small scale that separates stable accretion from unstable, fragmenting disc, which is crucial to our quantitative conclusion (note that they use the viscosity parameter $\alpha=0.05-0.15$ that is larger than our fiducial value of 0.01, which would yield a still smaller $r_{Q}$, see Equation 9). Thanks to that difference, we were able to conclude that, even without considering any feedback from the central SMBH, the SMBH during the starburst phase does not grow to anywhere close the observed final mass, because star formation can more favorably deplete the gas that may otherwise accrete to the SMBH, whereas they find SMBH masses to be too large even with substantial feedback (note that they use 10 times $L / c$ radiation pressure force assuming multiple scatterings of each converted FIR photon). It seems likely that their different conclusion may be due to a much higher accretion rate at their resolution scale, which we argue does not reflect the actual accretion onto the $\mathrm{SMBH}$, but rather the disc is unstable at that scale and mostly forms stars. As we have noted in the previous paragraph, observations indicate that the SMBH masses in the starburst phase appear to be smaller than the final values seen in quiescent elliptical galaxies by an order of magnitude, consistent with our conclusion. Substantially higher resolution (a factor of $\sim 100$ ) simulations may be necessary in order to realistically and more accurately simulate the intricate competition between accretion and star formation.

\section{A Comparison of Feedback Energetics Between Star Formation and SMBH}

Having shown the unlikelihood of substantially growing SMBH during the starburst phase, we now turn to a comparison of the energetics of SMBH and SF to show that, feedback from starburst itself should play the leading role in shutting down or quenching star formation, i.e., promptly sweeping away the final portion of the gas, where needed.

To avoid any apparent bias against SMBH or a possibly circular looking argument by the assertion that most of the SMBH growth takes place in the post-starburst phase (as we will show in $\S 4$ ), we shall for the moment generously assume that the entire SMBH growth occurs during the starburst phase, to maximize the energy output from the SMBH, when comparing the energetics from the SMBH and the starburst. In Table 1, under the assumptions that $\mathrm{M}_{\mathrm{BH}}: \mathrm{M}_{\mathrm{BG}}=2: 1000$, a Salpeter IMF for stars and a radiative efficiency of SMBH accretion of $10 \%$, energy output from both SF and SMBH in various forms are listed: (1) 
total radiation energy, (2) ionizing radiation, (3) X-ray radiation in $2-10 \mathrm{keV}$ band, (4) mechanical energy, which is supernova explosion energy for SF and broad absorption line (BAL) outflow for SMBH, respectively, and (5) radio jets. To obtain energy is ergs per $M_{\text {star }}$ formed, one just needs multiply each coefficient in Table 1 by $M_{\text {star }} c^{2}$, where $c$ is speed of light. The relevant references are Elvis et al. (1994) and Sazonov et al. (2004) for both $\epsilon_{\mathrm{BH}}(L L)$ and $\epsilon_{\mathrm{BH}}(2-10 \mathrm{keV})$, Ranalli et al. (2003) for $\epsilon_{*}(2-10 \mathrm{keV})$, Moe et al. (2009) and Dunn et al. (2010) for $\epsilon_{\mathrm{BH}}(\mathrm{BAL})$ and Allen et al. (2006) for $\epsilon_{\mathrm{BH}}(\mathrm{jet})$ (if one uses energy seen in the most powerful radio jet lobes and assumes that they are produced by the most massive $\mathrm{SMBH}$, a comparable value is obtained). The entry for the BAL energy is based on two cases and very uncertain, primarily due to lack of strong constraints on the location of the BAL and their covering factor.

It is evident that aside from the energy in the form of radio jets and hard X-rays, SF is at least competitive compared to SMBH. Heating due to hard X-rays from SMBH via metal line or Compton heating affects only the very central region surrounding the SMBH, not over the entire galaxy (Ciotti \& Ostriker 2007). Within the physical framework outlined here, most of the SMBH growth occurs post-starburst and radio jets occur at a still later stage in core elliptical galaxies, energy output (or momentum output derived from it) from $\mathrm{SF}$ in all relevant forms should dominate over that of SMBH. Our argument that radio jets occur at a later stage in galaxy evolution is not at present based on a physical model, but on empirical evidence. Observationally, it appears that all significant radio jets are launched in elliptical galaxies that have flat cores (Balmaverde \& Capetti 2006), with a very few exception that originated in disc galaxies (e.g., Evans et al. 1999; Ledlow et al. 2001) or S0's (e.g., Véron-Cetty \& Véron 2001). But none has been associated with elliptical galaxies with an inner powerlaw brightness profile slope. It has been plausibly argued that powerlaw elliptical galaxies are produced by gas-rich mergers (we adopt this scenario where a powerlaw elliptical galaxy is produced following each major gas-rich merger triggered starburst) (e.g., Faber et al. 1997), whereas core elliptical galaxies are produced later by dry mergers of two elliptical galaxies where the flat core is carved out by the merger of the two SMBHs via

Table 1.

\begin{tabular}{|c|c|c|c|}
\hline$\#$ & Form & $\mathrm{SF}$ & SMBH \\
\hline (1) & total radiation & $\epsilon_{*}(\mathrm{rad})=7 \times 10^{-3}$ & $\epsilon_{\mathrm{BH}}(\mathrm{rad})=2 \times 10^{-4}$ \\
\hline (2) & ionizing radiation $(\geq 13.6 \mathrm{eV})$ & $\epsilon_{*}(\mathrm{LL})=1.4 \times 10^{-4}$ & $\epsilon_{\mathrm{BH}}(\mathrm{LL})=3 \times 10^{-5}$ \\
\hline (3) & $\mathrm{X}$-ray $(2-10 \mathrm{keV})$ & $\epsilon_{*}(2-10 \mathrm{keV})=9 \times 10^{-8}$ & $\epsilon_{\mathrm{BH}}(2-10 \mathrm{keV})=5 \times 10^{-6}$ \\
\hline (4) & mechanical & $\epsilon_{*}(\mathrm{SN})=1 \times 10^{-5}$ & $\epsilon_{\mathrm{BH}}(\mathrm{BAL})=(0.2-2.8) \times 10^{-5}$ \\
\hline$(5)$ & radio jets & $\epsilon_{*}($ jet $)=0$ & $\epsilon_{\mathrm{BH}}($ jet $)=4 \times 10^{-5}$ \\
\hline
\end{tabular}


dynamical friction (e.g., Milosavljević \& Merritt 2001). Directly supporting this statement is the lack of radio jet in available observations of ULIRGs (e.g., Alexander et al. 2010), in agreement with other observations that indicate a significant time-delay between starburst and radio activities (e.g., Emonts et al. 2006). An independent, additional argument comes from the fact that radio jets are highly collimated and, for the most powerful ones that are energetically relevant, they appear to dissipate most of the energy at scales larger than that of the bulge region, suggesting that, even if one were to ignore the previous timing argument, the efficiency of heating by radio jets for the bulge region is likely low and at best non-uniform. Weaker radio feedback, observed almost exclusively in galaxies with an atmosphere of hot gas, may be able to steadily provide feedback energy but it is too weak to be energetically important. Besides, they appear to only operate in elliptical galaxies with hot atmospheres (e.g., Best et al. 2005).

The amount of supernova explosion energy that couples to the surrounding medium is $E_{\mathrm{SN}}=1 \times 10^{-5} M_{*} c^{2}$, which is exactly equivalent to $5 \times 10^{-3} \mathrm{M}_{\mathrm{BH}} c^{2}$ used in the influential simulations of Hopkins et al. (2006) with thermal AGN feedback, assuming $\mathrm{M}_{\mathrm{BH}}: \mathrm{M}_{\mathrm{BG}}=$ $2: 1000$. Because the energy output from supernovae is subject to less cooling than that from the AGN, since the former is at larger radii and lower densities than the latter, we expect that the amount of energy due to supernovae can at least as effectively as that proposed from AGN to drive the gas away. Thus, when most of the gas have formed into stars (i.e., the bulge is largely in place after $\sim 10^{7}-10^{8} \mathrm{yr}$ of starburst), the remaining gas should be blown away by collective supernova explosions and the starburst comes to a full stop, reminiscent of what is seen in the simulations of Hopkins et al. (2006) with AGN feedback. Detailed high-resolution simulations will be necessary, taking into account cooling and other physical processes, to ascertain the fraction of gas that is blown away. In short, the bulk of galactic winds is likely driven by stellar feedback from the starburst. Galactic winds are observed and casual connection between SF rate and wind fluxes has been firmly established (e.g., Heckman 2001; Weiner et al. 2009), lending strong observational support for the argument.

\section{Post-Starburst: Main Growth of SMBH with Self-Regulation}

The previous section ends when the starburst has swept away the remaining gas and ended itself. This section describes what happens next - the post-starburst period, the initial period of which is also known as $\mathrm{K}+\mathrm{A}$ galaxies.

The newly minted (future) bulge enters its "passive" evolutionary phase, as normally referred to. We would like to show that this is when most of the action for SMBH begins, fueled by recycled gas from aging low-to-intermediate mass stars. Since two-body relaxation time is much longer than the Hubble time, it is safe to assume that the stars formed in the inner region during the starburst phase remain roughly in place radially. Angular momentum 
relaxation may also be ignored for our purpose (e.g., Rauch \& Tremaine 1996). However, the stellar distribution in the inner region that initially formed on a disc probably has vertically thickened substantially and we will assume that they no longer substantially contribute to local gravity on the gas disc (within the thickness of the assumed thin gas disc) subsequently formed from returned stellar gas. Because stars in the inner regions are already mostly rotationally supported, the shedded gas rains almost "straight down" to land at a location that their specific angular momentum allows, to form a disc. Obviously, going out radially, the rotational support lessens and star formation may occur in a 3-d fashion. But that does not alter our argument about what happened at small radii. The orientation of the disc is approximately the same as the previous disc out of which stars in the inner regions were formed, since the overall angular momentum distribution of stars has not much changed in the absence of any subsequent intrusions. The most important difference of this new accretion disc, compared to the disc formed during the starburst phase, is that this new disc starts with almost no material and surface density increases with time gradually on the timescale of hundreds of megayears to gigayear.

To have a better gauge how the results obtained depend on the assumed inner density slope, instead of assuming a Mestel disc as is done in $\S 3$ here we present a more general case assuming the inner density profile of the form

$$
\Sigma_{g}(r)=\Sigma_{0}\left(\frac{r}{r_{0}}\right)^{-n}
$$

where $n \sim[0.5,1]$ (e.g., Faber et al. 1997; Kormendy et al. 2009). For this case Equation (8) is modified, taking into account the gradual change of the gas disc surface density with time, to be

$$
\begin{aligned}
r_{Q}= & \frac{1}{\left(\pi(3 \pi)^{1 / 2}\right)^{4 / 3(3-2 n)}}\left(\frac{\dot{M}}{M}\right)^{2 / 3(3-2 n)} \\
& \left(f_{\mathrm{rec}} f_{g}\right)^{-2 /(3-2 n)} \alpha^{-2 / 3(3-2 n)} G^{-1 / 3(3-2 n)} M^{5 / 3(3-2 n)} \Sigma_{0}^{-2 /(3-2 n)} r_{0}^{-2 n /(3-2 n)}
\end{aligned}
$$

where $f_{\text {rec }}$ is the total fractional stellar mass that recycles back to ISM and $f_{g}(t)$ the fraction of recycled gas that has returned by time $t$ (out of the fraction $f_{\text {rec }}$ ). The process of SMBH accretion in this case goes as follows. The $\mathrm{SMBH}$ will accrete all the gas within its Bondi radius $r_{B}$ over some period of time, as long as $r_{Q} \geq r_{B}$, where $r_{B}$ is defined as

$$
r_{B} \equiv \frac{G \mathrm{M}_{\mathrm{BH}}}{\sigma_{n}^{2}},
$$

with $\sigma_{n}$ being the velocity dispersion of the inner region of the bulge $(r \leq 20 \mathrm{pc}$ or so for $\left.\mathrm{M}_{\mathrm{BH}}=10^{8} \mathrm{M}_{\odot}\right)$. For the moment we ignore any feedback effect from the SMBH. Since $r_{B}$ grows with time and $r_{Q}$ decreases with time with increasing $f_{g}$ for $r>r_{B}$ that has been 
accumulating gas, the condition $r_{Q} \geq r_{B}$ may be violated at some time $t$, at which point the $\mathrm{SMBH}$ is cut off gas supply at its Bondi radius and the SMBH will subsequently grow by consuming the final patch of gas on the disc within its Bondi radius. Before the condition $r_{Q} \geq r_{B}$ is reached, the recycled gas that has landed outside (time varying) $r_{B}$ continues to accumulate (some of the accumulated gas possibly forms stars). Using Equations (11 12) we find the turning point $r_{Q}=r_{B}$ is reached when

$$
f_{\text {rec }} f_{g}=\frac{2-n}{2}
$$

with the disc mass within $r_{Q}=r_{B}$, i.e., SMBH mass, being

$$
M_{F}=\frac{3(2-n)^{3}}{8}\left(\frac{\dot{M}}{M}\right)^{-1} \frac{\alpha \sigma_{n}^{3}}{G} .
$$

From Equation (13) we see that $(2-n) /\left(2 f_{\text {rec }}\right)>1$ for $n=[0.5-1]$. Thus, we simply correct Equation (14) by a factor of $2 f_{\text {rec }} /(2-n)$ to finally arrive at

$$
\begin{aligned}
\mathrm{M}_{\mathrm{BH}} & =\frac{3(2-n)^{2}}{4}\left(\frac{\dot{M}}{M}\right)^{-1} \frac{f_{\mathrm{rec}} \alpha \sigma_{n}^{3}}{G} \\
& =1.9 \times 10^{8}(2-n)^{2} \alpha_{0.01} l_{E}^{-1} \epsilon_{0.1}\left(\frac{\sigma_{n}}{200 \mathrm{~km} / \mathrm{s}}\right)^{3} \mathrm{M}_{\odot},
\end{aligned}
$$

with the radius when $r_{Q}=r_{B}=r_{B Q}$ being:

$$
r_{B Q}=34(2-n)^{3} \alpha_{0.01} l_{E}^{-1} \epsilon_{0.1}\left(\frac{\sigma_{n}}{200 \mathrm{~km} / \mathrm{s}}\right) \text { pc. }
$$

Equations 15, 16 suggest that the SMBH accreted the recycled gas at $r \leq 20\left(\mathrm{M}_{\mathrm{BH}} / 10^{8} \mathrm{M}_{\odot}\right) \mathrm{pc}$ or so for $l_{E} \sim 1$; it could be substantially larger for smaller $l_{E}$. The reason that the accretable mass is so much larger during this period than the starburst phase is because the accretion disc in this period is replenished continuously at a moderate rate such that it is stable within a much large radius than the case of sturburst phase with a much thicker (surface density-wise) disk. Equation (15) resembles the observed $\mathrm{M}_{\mathrm{BH}}-\sigma$ relation (Tremaine et al. 2002). We argue the resemblance is deceptive, in a general sense, because it hinges on a value of $\alpha \sim 0.01$ or so and $l_{E} \sim 1$. As we mentioned earlier, the currently allowed value of $\alpha$ could range from $10^{-4}$ to 1 and at the moment we do not know what value nature has picked to grow her SMBHs. In light of this situation, using Equation 15 to declare victory is premature. However, Equation (15) does suggest that there is enough material and time to grow the SMBH to the observed value during the post-starburst phase. This is in stark contrast with the starburst phase when there is not enough accretable matter even if one pushes the viscosity value to the limit (see Equation 9). 


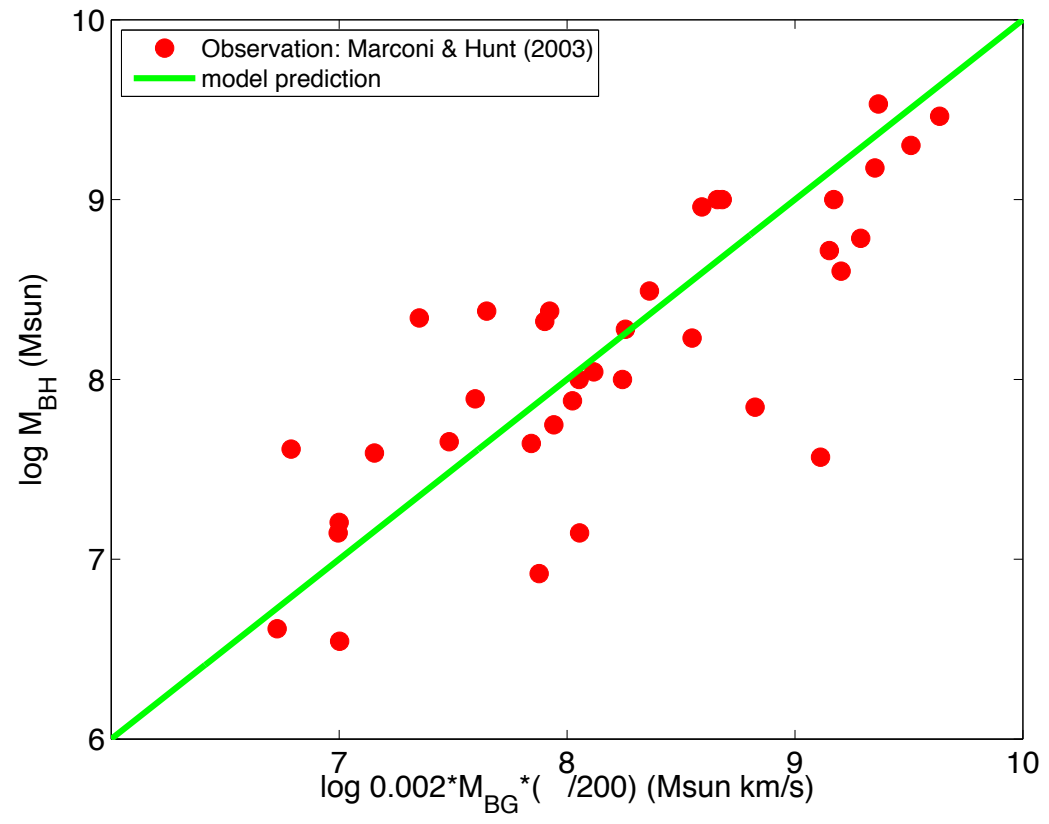

Fig. 1. - The circles are data from Marconi \& Hunt (2003). The solid line is predicted by Equation 17 using $A=1$ (see Equation 18).

A scenario where the allowed range of viscosity value is limited to one side, i.e., $\alpha$ is allowed to have values greater than say 0.01 , is much less fine tuned. In this case, some self-regulation for the SMBH growth will be necessary. This self-regulation for the SMBH growth is indeed achievable during the post-starburst phase, as we will now describe. The total amount of radial momentum that radiation pressure of the SMBH may exert on the surrounding gas is $\epsilon c \mathrm{M}_{\mathrm{BH}}$ (this is likely a lower bound, neglecting the possibility of multiple scatterings of photons in the optically thick regime). Equating $\epsilon \beta c \mathrm{M}_{\mathrm{BH}}$ to $f_{\mathrm{rec}} \mathrm{M}_{\mathrm{BG}}\left(1-f_{*}\right) v_{\mathrm{esc}}$ (that is the momentum of the driven-way gas escaping the galaxy) gives

$$
\frac{\mathrm{M}_{\mathrm{BH}}}{\mathrm{M}_{\mathrm{BG}}}=\frac{f_{\mathrm{rec}} v_{\mathrm{esc}}}{\epsilon \beta c} \frac{\left(1-f_{*}\right)}{\left(1+f_{\mathrm{rec}} f_{*}\right)}=A \frac{2}{1000} \sigma_{200},
$$

where $A$ is

$$
A=\frac{\left(f_{\text {esc }} / 0.15\right)\left(1-f_{*}\right)}{\left(1+\left(f_{\mathrm{rec}} / 0.15\right) f_{*}\right) \beta \eta \epsilon_{0.1}} \frac{v_{\mathrm{esc}}}{2 \sigma},
$$

where $f_{*}$ is the fraction of recycled gas that subsequently re-formed into stars and $v_{\text {esc }}$ is escape velocity [for an isothermal sphere truncated at virial radius $r_{v}, v_{\text {esc }}(r) / 2 \sigma=(1+$ $\left.\ln \left(1+r_{v} / r\right)\right)^{1 / 2}$ at radius $\left.r\right] ; \beta$ is the fractional solid angle that absorbs the radiation from 
the SMBH; the term $\left(1+f_{\text {esc }} f_{*}\right)$ takes into account additional stars added to the bulge stellar mass formed from the recycled gas. Of the parameters in Equation 18, $f_{\text {esc }}=0.15$ is reasonable taking into account that about the half of mass return occuring at early times by type II supernovae can escape without additional aid; radiative efficiency of $\epsilon=0.1 \epsilon_{0.1}$ is consistent with observations ( $\mathrm{Yu} \&$ Tremaine 2002; Marconi et al. 2004); some fraction of the recycled gas forming into stars is probably unavoidable, since some gas with column density greater than Compton column will slip through radiation pressure (see discussion below); $f_{*}$ also includes the (possibly very large) amount of gas at large radii that would not have accreted onto the SMBH in the first place even in the absence of any feedback (e.g., molecular clouds on the Galactic disk are not being fed to the Galactic center SMBH in a consistent fashion); the factor $\eta$ (greater than one) takes into account additional stars that are not formed from the starburst event. Overall, considering all these balancing factors, a value of $A$ of order unity seems quite plausible. Figure 1 plots the relation between $\mathrm{M}_{\mathrm{BH}}$ and $\sigma \mathrm{M}_{\mathrm{BG}}$ predicted by Equation 17 using $A=1$. It is clear that it provides a very good fit to the observed data. A similar scaling relation as Equation 17 was derived based on a different, radio jet feedback mechanism (Soker \& Meiron 2010).

A similar scenario of linear momentum feedback from AGN radiation pressure has been considered by Silk \& Nusser (2010) to possibly produce the observed $M_{B H}-M_{B G}$ relation during the starburst phase but they conclude that the radiation pressure is insufficient by an order of magnitude to be able to blow the unwanted gas away. The magnitude of the radiation pressure and escape velocity requirement considered here are the same as theirs. The difference is that here the amount of gas that need to be regulated in the post-starburst phase is nearly a factor of 10 lower and further allowance for star formation from the recycled gas make possible that the radiation pressure from the central AGN may be adequate to self-regulate the SMBH growth so as not to overgrow it.

We note that Equation 17 would work without much variation if the gas that is blown away is uniformly distributed. The recycled gas is expected to be non-uniform. Even if it were uniform initially, thermal instabilities likely make the distribution non-uniform. Given that, we elaborate further on Equations 1718 and the physical processes of radiation pressure driven winds. Some distinction may be made between about $1 / 3$ of the total solid angle where UV and other photons are directly seen from AGN and the other $\beta \sim 2 / 3$ of the solid angle that has a nearly Compton thick or thicker obscuring screen, most of which probably stems from the so-called molecular torus (e.g., Risaliti et al. 1999). For every $\Delta M_{\text {acc }}$ of mass accreted, roughly $\epsilon c / v_{\text {esc }} \Delta M_{a c c}=100 \epsilon_{0.1}\left(v_{e s c} / 300 \mathrm{~km} / \mathrm{s}\right)^{-1} \Delta M_{a c c}$ of mass that rain down by aging stars could be driven away by the radiation momentum from the AGN. In the 1/3 opening solid angle some portion of the radiation pressure driven winds will be accelerated to high velocities, perhaps in a fashion similar to what is seen in simulations (e.g., Kurosawa \& Proga 2009), observationally manifested as broad emission or absorption lines as well as outflows seen in narrow lines (e.g., Crenshaw et al. 2003; Greene et al. 2011). 
A significant fraction of the material may be accumulated in the remaining $\beta \sim 2 / 3$ of the solid angle (i.e., Type 2 AGNs), including recycled gas that comes from the other $1 / 3$ solid angle that is too heavy to be accelerated away "on the fly" by the radiation pressure. In this $2 / 3$ of the solid angle, high velocity winds radially exterior to the molecular torus is unlikely given the heaviness (i.e., low opacity) of the molecular torus. We discuss some of the physics here. To gain a more quantitative understanding, a look at some observed properties of the torus is instructive. Jaffe et al. (2004) measured the radius and height of the molecular torus of NGC 1068 to be $1.7 \mathrm{pc}$ and $2.1 \mathrm{pc}$, respectively. The mass of the SMBH in NGC 1068 is $(8.3 \pm 0.3) \times 10^{6} \mathrm{M}_{\odot}$ (e.g., Marconi \& Hunt 2003). If we extrapolate to a $10^{8} \mathrm{M}_{\odot} \mathrm{SMBH}$ assuming that the location and height of the molecular torus is proportional to the SMBH mass, we have a surface area of the torus equal to $3200 \mathrm{pc}^{2}$ at a SMBH-centric radius of 20 pc. If we assume that the column density of the molecular torus is $10^{24} \mathrm{~cm}^{-2}$ (e.g., Risaliti et al. 1999), its total mass is then $2 \times 10^{7} \mathrm{M}_{\odot}$. The dynamical time at $20 \mathrm{pc}$ is $10^{5}$ yrs. A SMBH of mass $10^{8} \mathrm{M}_{\odot}$ accreting at Eddington rate would grow a mass of $\sim 10^{5} \mathrm{M}_{\odot}$ in $10^{5} \mathrm{yrs}$, while the overall rate of gas return would be $\sim 2 \times 10^{7} \mathrm{M}_{\odot}$ over the entire bulge during that period. Thus, the abundant gas supply rate suggests that the necessary (not sufficient) condition for a near "steady" state is met such that the molecular torus may be kept roughly invariant with time, with the rate of driven-away gas by radiation pressure plus that of gas forming into stars equal to the rate of gas return from aging stars.

Given the short star-formation timescale of the very dense gas in the molecular torus, it would be unavoidable that star formation should occur there (as well as some regions exterior to it). This "lightens up" the torus to the extent that it may be pushed away by the radiation pressure, when the condition that the deposited radiation momentum divided by the accumulated mass exceeds the escape velocity (assuming, in the absence of radiation pressure, the torus would just be in a bound circular orbit). In this sense the radiation momentum from the SMBH serves to retard gas supply to accretion from the torus to let SF take over to have it mostly depleted. In combination with the analysis in the preceding paragraph, it seems physically plausible that radiation pressure and depletion of gas by star formation is able to jointly reduce and regulate the amount of gas that feeds the central SMBH. Given that the overall margin, in an "on average" sense, is quite thin (i.e., $A \sim 1$ in Equation 18, it is likely that there are significant variations in $A$, perhaps up to a factor of a few.

In the 1-d simulations of Ciotti \& Ostriker (2007) for an elliptical galaxy, the SMBH growth appear to be intermittent. The intermittency in their simulations was caused by a hot X-ray heated bubble that prevents continued gas accretion, until it bursts, which is then followed by another accretion episode, and so on. We suggest that Rayleigh-Taylor instability on the shell enclosing the X-ray bubble may prevent the X-ray bubble from inflating, as hinted by recent 2 -d simulations of Novak et al. (2010). It it reasonable to assume that shell fragmentation in three-dimension is still more pronounced to allow continued deflation 
of a notional X-ray bubble. Observationally, the lack of significant X-ray emission from circumnuclear region in powerlaw elliptical galaxies host AGNs, which we argue are the post-starburst galaxies we consider here, supports the picture that the hot bubble is not robust (e.g., Pellegrini 2005). In the absence of a hot X-ray bubble guarding the SMBH, we suggest that the recycled gas from aging stars is able to reach the disc and the accretion, with self-regulation argued above, is quasi-steady without major flares of magnitude seen in 1-d simulations. As we will show later, a steady declining accretion rate proportional to the gas return rate provides a much better match to at least two observations: (1) the observed early-type host galaxies of AGNs are mostly in the green valley of the galaxy color-luminosity diagram with a small fraction in the red sequence (§5.2) (e.g., Salim et al. 2007; Silverman et al. 2008; Hickox et al. 2009; Schawinski et al. 2010), but very few in the blue cloud, which would have been the case if AGN flares are accompanied by starbursts (Ciotti \& Ostriker 2007); (2) the observed AGN accretion rate for early-type galaxies in the local universe displays a powerlaw distribution with the amplitude and decay rate (Kauffmann \& Heckman 2009 ) that is expected from the non-flare scenario that is proposed here. This indicates that bursty AGN accretion, while quite possible and sometimes perhaps unavoidable, is probably not the dominant mode. It is currently a challenge but will be of great value to carry out 3-d high-resolution simulations to more accurately quantify this outcome.

\section{Model Predictions and Discussion}

We have presented a physically motivated picture for the coevolution of galaxies and SMBH starting with a triggered starburst. Let us now summarize the entire evolution in $\S 5.1$ and then give an incomplete list of implications and predictions in $§ 6.2-6.9$ to be qualitatively compared/verified with observations.

\subsection{Three Distinct Periods of Coevolution of Galaxies and SMBH}

From the onset of a significant central starburst to becoming a quiescent bulge there are three distinct periods, as summarized in Figure 2 for an example merger of two gas-rich spirals each of mass $\sim 10^{12} \mathrm{M}_{\odot}$ that eventually becomes a powerlaw elliptical galaxy of velocity dispersion of $200 \mathrm{~km} / \mathrm{s}$. We stress that the trigger event is not limited to major mergers. This three-stage scenario is not new and its successes with respect many observations have been discussed previously (e.g., Granato et al. 2004, 2006, Cirasuolo et al. 2005; Lapi et al. 2006; Lamastra et al. 2010). The new theoretical element here is the primary growth of $\mathrm{SMBH}$ in the post starburst phase, which is reflected in the color and other properies of AGN hosts and we will show is in remarkable accord with latest observations, in contrast to the conventional scenario where SMBH growth primarily occurs during the starburst phase. 
The time boundaries between difference consecutive phases (three ovals) are approximate (uncertain to a factor of at least a few). Given the complexity and variety of starburst trigger events, one should expect significant variations from case to case. The expected consequences or predictions of this model are in many ways different from and often opposite to those of models that invoke AGN feedback to shut down both starburst and AGN activities (e.g, Silk \& Rees 1998; Hopkins et al. 2006). A new and in some way perhaps the most fundamental finding of this work is that the SMBH does not grow during the starburst phase as much as previously thought, required in AGN-feedback based models, despite the obvious condition that there is a lot of gas being "jammed" into the central region; this is different from almost all previous work (e.g, Silk \& Rees 1998; Hopkins et al. 2006; DeBuhr et al. 2010) that either need to advocate very strong SMBH feedback or appear to overgrow the SMBH.

The idea of feeding the SMBH with recycled stellar material in the post-starburst phase is not new (e.g., Norman \& Scoville 1988; Ciotti \& Ostriker 2007) and we inherit most of the already known elements from prior work, including gas return rate and the likelihood of continued star formation. Our analysis shows the likelihood that the SMBH may be fed too much in the post-starburst period in the absence of feedback from the SMBH, in dramatic contrast with the starburst phase when SMBH feedback is insufficient. While energy feedback from the SMBH certainly plays a role, we show that the more robust momentum feedback from SMBH radiation pressure can play a critical role in regulating SMBH growth, not necessarily only by blowing powerful winds, but rather, in combination, by also pushing away thus retarding accretion of unwanted (by SMBH) gas to be instead consumed by star formation. While our analysis may have captured some of the essential physics in terms of accretion and star formation demarcation, to more realistically model the complex accretion and star formation dynamics, much higher resolution 3-d radiation hydrodynamic simulations will be required and will be of tremendous value.

The "size" of the starburst depends on the "size" of the triggering event, with at least some fraction of ULIRGs and SMGs due to major mergers of massive gas-rich gas. However, irrespective of the size of the starburst event, the time scales involved, being largely due to physics of stellar interior and accretion time scale, remain the same.

(1) "Starburst Period": this phase is triggered by some event. The SMBH grows modestly during this period to possibly attain a mass that is up to order ten percent of its final mass. This phase lasts about $10^{7}-10^{8}$ yrs for typical starbursts and the host galaxies during this phase are in the blue cloud in the luminosity-color diagram. The feedback energy/momentum from the starburst, i.e., supernovae, drives the last patch of gas away and shuts down star formation, if needed. In other words, the starburst is self-regulated, not by the central AGN during this period.

(2) "SMBH Prime Period": several hundred million years after the end of the starburst, aging low-to-intermediate mass stars, now in their post-main-sequence phases, start to return a 
substantial fraction of their stellar mass to the ISM. The SMBH accretion is fueled by this recycled gas lasting for order of gigayear. The growth of SMBH is self-regulated, readily provided by the radiation pressure from the AGN. The host galaxies during this period start out light-blue or in the "green valley" and migrate to the "red sequence". Because the rate of gas return from stars diminishes with time and SMBH mass grows, the Eddington ratio of the SMBH decreases with time. The SMBH growth is synchronous with star formation from recycled gas during this period. The accompanying star formation rate may also be substantial but typically does not constitute a starburst during this period. The entire duration of this phase depends sensitively on the lower cutoff mass of the initial mass function (IMF) - a sensitive and powerful prediction of this model.

(3) "Quiescent Bulge": several gigayears after the end of the starburst the bulge is now truly red and dead - gas return rate is now negligible so both accretion to the central SMBH and residual star formation have ceased. It is possible that a disk is grown later around the bulge. The feeding of the central SMBH in the bulge of spiral galaxy during this period is no longer by overhead material from aging stars, rather by occasional objects that happen to be on some plunging orbits to be disrupted by the SMBH and form a short-lived accretion disc. Candidate objects may include molecular clouds, some tidally disruptable stars or gas streams. Significant disturbances or torques, such as minor mergers and galactic bars, could provide the necessary drivers for some more consistent accretion events. How is a red and dead bulge with a hot atmosphere able to remain star-formation-free? This is a major topic on its own right and beyond the scope of the current paper, but will be addressed in a future paper.

\subsection{Some "Obvious" Implications of the Model}

There are some unambiguous discriminating signatures of this model that already can be directly "read off" Figure 2. We highlight several here.

(1) Starburst and AGN growth are not coeval in this model. AGN does not regulate the starburst, consistent with observations (e.g., Schawinski et al. 2009; Kaviraj 2009). AGN activities is expected to outlive the starburst, in agreement with observations (e.g., Georgakakis et al. 2008). These predictions are opposite to those of models that invoke AGN feedback as the primary regulating agent.

(2) The apparent requirement of a rapid migration of early-type galaxies from the blue cloud to the red sequence, in order to produce a bimodal distribution in color (e.g., Blanton

et al. 2003), is primarily due to the prompt shutdown of SF by stars (i.e., supernovae) at the end of the starburst phase; there is no need to invoke other ingredients, consistent with observations (e.g., Kaviraj et al. 2010). Observationally, there is no evidence that the 
presence of an AGN is related to quenching of star formation or the color transformation of galaxies (e.g., Aird et al. 2012). This prediction is different from that of models that invoke AGN feedback to quench star formation.

(3) AGN activities in ongoing starburst galaxies, i.e., buried AGN activities, are not expected to be dominant in this model, in agreement with observations (e.g., Genzel et al. 1998; Ivison et al. 2000; Ptak et al. 2003; Ivison et al. 2004; Alexander et al. 2005a.b; Schweitzer et al. 2006; Kawakatu et al. 2006; Alexander et al. 2008; Veilleux et al. 2009). Note that the above statement is not inconsistent with AGN/QSOs being associated with galaxies in the process of merging, which may enhance accretion activities in the involved (yet to merge) galaxies (e.g, Bahcall et al. 1997; Hennawi et al. 2010; Smith et al. 2010).

(4) The most luminous quasars that accrete with high Eddington ratios occur order of $100 \mathrm{Myr}$ after the end of the starburst. They may contain substantially more merger signatures, which appears to be indicated by observations (e.g., Bennert et al. 2008). If one were to identify a population in-between ULIRGs and more regular QSO hosts in terms of spectral properties, they should show some more signs of tidal interactions that are yet to fully settle since the starburst, also consistent with observations (e.g., Canalizo \& Stockton 2001).

(5) Low Eddington ratio AGNs that are expected to last order of Gyr are not expected to show a close linkage to major disturbances that trigger the starburst (e.g., mergers), since possible signatures of the trigger merger event have largely been erased over time, consistent with observations (e.g., Grogin et al. 2005; Cisternas et al. 2011). Thus, one does not expect to see merger signatures to be associated with moderate-luminosity AGNs, which is in contrast with AGN feedback based models where most of the moderate luminosity AGNs are expected to coincide with starburst.

(6) While the green-valley morphologically early-type galaxies that host AGN is the evolutionary link between starburst galaxies (in the blue cloud) and the red elliptical galaxies (on the red sequence), it is useful to distinguish between them and the other class of green galaxies that simply continuously form a modest amount of stars (such as our own Galaxy). The former are chronologically immediate successors to starburst galaxies and should be in early-type galaxies, strongly supported by observations (e.g., Salim et al. 2007; Silverman et al. 2008; Hickox et al. 2009; Schawinski et al. 2010), whereas the latter are not a chronologically intermediate class between the blue cloud and the red sequence. The total green galaxy population will be the sum of these two different morphological types, with some obvious implications, such as green galaxies having mixed morphological types with limited merger signatures, consistent with observations (e.g., Mendez et al. 2011). This prediction is in contrast with AGN feedback based models where most AGN hosts are expected to coincide with starburst and a small fraction, mostly the most luminous AGNs (occuring near the end of the starburst phase), is expected to have matured early-type morphologies. 
(7) While the early-type AGN host galaxies may have similar morphologies as and will eventually evolve to inactive elliptical galaxies, the former should have much bluer colors than the latter, consistent with observations (e.g., Sánchez et al. 2004). The basic morphological properties of the host galaxies of the most luminous quasars, corresponding to the most massive SMBHs in the prime growth phase should resemble those of giant elliptical galaxies, consistent with observations (e.g., Dunlop et al. 2003).

(8) Because of the expected rate of gas return ( $\propto t^{-1.3}$ on gigayear scales $)$ to which both SMBH accretion and star formation are proportional and because more powerful AGN accretion occurs closer in time to the preceding starburst, it is expected that more powerful AGNs are hosted by early-type galaxies with younger mean stellar ages, consistent with observations (e.g., Kauffmann et al. 2003; Jahnke et al. 2004).

(9) The accompanying star formation rate of elliptical galaxies may be quite substantial, on the order of $\sim(5-10)\left(M_{*} / 10^{11} \mathrm{M}_{\odot}\right)(t / 1 \mathrm{Gyr})^{-1.3} \mathrm{M}_{\odot} \mathrm{yr}^{-1}$. Thus, while most AGN host galaxies have left the blue cloud, a significant fraction of them, especially those hosting luminous AGNs, should still have substantial SFR, consistent with observations (e.g., Silverman et al. 2009; Shi et al. 2009). It is expected that the incidence of star formation signatures (e.g., dust) in the nuclear region should correlate positively with AGN activities for elliptical galaxies, because the strengths of both are proportional to the gas return rate, consistent with observations (e.g., Simões Lopes et al. 2007). These predictions are opposite to AGN feedback based models where star formation is expected to be completely quenched after AGN feedback clears the gas out.

\subsection{Origin of Two AGN Accretion Regimes}

Kauffmann \& Heckman (2009) presented an insightful observational result of two distinct regimes of black hole growth in nearby galaxies along with its apparent implications. They find that star-forming galaxies display a lognormal distribution of Eddington ratios; their interpretation is that in this regime accretion on to the SMBH is not limited by the supply of gas but by feedback processes that are intrinsic to the SMBH itself. Our model provides the following alternative interpretation for this phenomenon: this lognormal distribution merely reflects two random processes at work: (1) the amount of gas that landed on the stable accretion disc to provide accretion to the SMBH during the starburst phase depends on many "random" variables of the triggering event (in the case of a merger, such as merging orbit inclination, velocity, spin alignment, etc), and (2) observations catch a random moment during the accretion of this gas. Central theorem should then give rise to a lognormal distribution. Another class of possible triggering events for SMBH accretion in star-forming galaxies (e.g., dormant SMBH in the bulge of disk galaxies) is stochastic feeding due to some random events, which should also follow a lognormal distribution. 
Separately, they find that galaxies with old stellar populations is characterized by a power-law distribution function of Eddington ratios and the AGN accretion rate is about $0.3-1 \%$ of the gas return gas from recycling. In our model the expect accretion rate is expected to be $\mathrm{M}_{\mathrm{BH}} /\left(f_{\mathrm{rec}} \mathrm{M}_{\mathrm{BG}}\right)=1.3 \times 10^{-2} A \epsilon_{0.1}^{-1} \sigma_{200}$. This expected relation between $\mathrm{SMBH}$ accretion rate and gas return rate is remarkably close to their observed value. As Kauffmann \& Heckman (2009) already pointed out, the powerlaw distribution is consistent with the recycling gas return rate $\propto t^{-1.3}$ (Mathews 1989). This is a strong support for the proposed model here.

\subsection{Initial Mass Function and AGN Accretion History}

Because the least massive stars live the longest, the cutoff mass of the initial stellar mass function (IMF) plays an important role in shaping the evolution on longer time scales of $\geq 1$ Gyr. For example, a $0.92 \mathrm{M}_{\odot}$ star (solar metallicity) has a lifetime of $10 \mathrm{Gyr}$, whereas a $1.4 \mathrm{M}_{\odot}$ only lives $\sim 2 \mathrm{Gyr}$. Thus, the duration of the "SMBH Prime Period" depends sensitively on the lower mass cutoff of the IMF. Figure 3 shows several cases of the evolution of the SMBH growth tracks. It shows that the evolution and duration of SMBH growth in the post-starburst phase depend sensitively on the low mass cutoff of the IMF. We see that for a cutoff mass of $0.92 \mathrm{M}_{\odot}$ the $\mathrm{SMBH}$ spends about $100 \mathrm{Myr}$ accreting at Eddington limit when its mass is up to about $10 \%$ of its final mass and a significant period ( $\geq 1 \mathrm{Gyr}$ ) at less than $1 \%$ of the Eddington rate, and most of the time at about $0.1 \%$ of the Eddington rate when its mass approaches its final mass. On the other hand, with a mass cutoff of $1.4 \mathrm{M}_{\odot}$ the entire SMBH accretion shortens to $2 \mathrm{Gyr}$ and does not extend below $10^{-2}$ Eddington rate. Since not all elliptical galaxies at present time are observed to accrete at $0.1 \%$ of the Eddington rate, this already suggests that a higher than $0.92 \mathrm{M}_{\odot}$ cutoff mass in the IMF may be required. Presently there is circumstantial evidence for massive star formation in galactic centers, including our own Galaxy (e.g., Lu et al. 2009) and M31 (e.g., Bender et al. 2005). Given the very sensitive dependence of stellar lifetime on stellar mass, careful considerations along this line may prove to be very powerful in placing constraints on the low-mass cutoff in the IMF as well as testing this model. Detailed comparisons between theoretical prediction

with observational data in terms of the AGN luminosity-mass plane (e.g., Steinhardt \& Elvis 2010), the Eddington ratio range (e.g., Woo \& Urry 2002), AGN ages at different redshifts (e.g., Martini 2004) or at different luminosities (e.g., Adelberger et al. 2005) should also prove very powerful in constraining the IMF. We note that our assumption used to derive the light curves in Figure 3 is extremely simplistic and therefore we do not expect that they provide satisfactory matches to observations. It is called for that additional ingredients be included to account for, e.g., variations in stellar distribution, possible variations of IMF as a function of local star formation conditions, dependence of initial seed SMBH mass on galaxy model, etc, in order to have a more encompassing analysis. We shall carry out a more detailed 
analysis with additional parameters in a future study, especially when measurements of both $\mathrm{SMBH}$ masses and accretion rates become significantly more precise for a large sample of active galaxies.

\subsection{Super-Solar Metallicity of Accreting Gas}

One clear implication is that the accretion gas, being shedded from aging stars, should be very metal rich with supersolar metallicity, in agreement with observations (e.g. Hamann \& Ferland 1993), especially to explain super-solar N/He ratio (e.g., Hamann \& Ferland 1999). This is because nitrogen is believed to be secondary nature, where its abundanace scales quadratically with metallicity. The recycled gas that is feeding the SMBH in our model fits the bill most naturally. In addition, the metallicity of accretion gas is not expected to depend on redshift, being intrinsic to stellar evolution, consistent with all accreting gas being very metal rich at all redshifts, including the highest redshift SDSS quasars (e.g., Fan et al. 2006).

\subsection{Relative Cosmic Evolution Between Starburst Galaxies and AGN}

Given the modest amount of time delay (several 100Myrs) between the starburst phase and the SMBH prime growth phase, it is unsurprising that one should expect to see nearly synchronous evolution between the starburst and SMBH growth on longer, cosmic time scales, consistent with observations (e.g., Boyle et al. 1988; Nandra et al. 2005).

In the context of the observed cosmic downsizing phenomenon, the downsizing of galaxies (e.g., Cowie et al. 1996, Treu et al. 2005) should thus be closely followed by downsizing of AGNs (e.g., Barger et al. 2005; Hasinger et al. 2005). There is, however, a very important difference between the two classes in post peak activities, predicted in this model. For starburst the shutdown time scale is expected to be about $\sim 100 \mathrm{Myrs}$, whereas for moderateluminosity AGNs (i.e., Eddington ratio $\sim 10^{-3}$ ) the decay time scale is of order of $\sim 1$ Gyrs. With a deep AGN survey that is capable of subdividing early-type galaxies in terms of their masses, one should be able to differentiate between the downturn time of starburst galaxies

and that of AGNs hosted by elliptical galaxies at a fixed mass. This prediction would be a strong differentiator between this model and AGN-based feedback models. 


\subsection{AGN Broad Emission and Absorption Lines}

Some of the overhead material raining down onto SMBH accretion disc from recycled gas from aging low-to-intermediate mass stars provides the material observed as broad emission lines (BEL) and broad absorption lines (BAL). When some of this gas, probably in the form of some discrete clouds, reaches the inner region of the the SMBH (at $r \leq 10^{2} r_{s}$, where $r_{s}$ is Schwarzschild radius), the clouds will be accelerated by radiation pressure, likely through some absorption lines, to velocities up to 0.1c. These clouds will be the observed BEL and BAL. The fact that only $15-20 \%$ of type I AGN to have BAL may be indicative of the discrete nature of the clouds, not unexpected from discrete stellar remnants or from cooling instabilities.

An advantage of this overhead material is that it naturally provides gas clouds that are presumably to be some $\geq 50^{\circ}$ off the equatorial plane, in order not to be obscured by the molecular torus (there are of course BEL and BAL gas clouds at smaller angles but they are not seen directly). In this model we do not need any additional pressure force to lift the gas off the accretion disc - some of the raining down gas clouds from aging stars will be launched outwards before they reach the disc, physics of which is well known (e.g., Murray et al. 1995).

\subsection{Evolution of SMBH Mass Relative to Bulge Mass}

Massive elliptical galaxies appear to have increased their masses by $30-100 \%$ in the last 7 Gyr (e.g., Brown et al. 2008). The growth of the elliptical mass is not expected to be always accompanied by corresponding growth in the mass of the central SMBH. For example, merger of a spiral galaxy without a significant SMBH and an elliptical galaxy would make the final SMBH appear less massive. Given the dependence of $\mathrm{M}_{\mathrm{BH}} / \mathrm{M}_{\mathrm{BG}} \propto \sigma \propto(1+z)^{1 / 2}$ predicted in this model, we predict that the $\mathrm{M}_{\mathrm{BH}} / \mathrm{M}_{\mathrm{BG}}$ relation should evolve with redshift stronger than $(1+z)^{1 / 2}$ for quiescent elliptical galaxies.

\subsection{On Relation between SMBHs and Pseudo-bulges}

It is useful to add a note on the difference between classic bulges and pseudo-bulges (Kormendy \& Kennicutt 2004) with respect to the central SMBHs in this model. The relation derived, Equations (17, 18), that matches the observed $\mathrm{M}_{\mathrm{BH}}-\mathrm{M}_{\mathrm{BG}}$ relation is dependent on the abundant supply of recycled gas in the inner region. Given the sufficient gas supply from recycled gas, the feedback from the SMBH then can regulate its own growth. This essential ingredient of sufficient gas supply is consistent with the observed inner slope 
of classic bulges (e.g., Faber et al. 1997; Kormendy et al.|2009), as we have shown.

The situation would be very different, if star formation is not as centrally concentrated as in classic bulges, for example, in rings (Kormendy \& Kennicutt 2004, and references therein) of high angular momentum with a hollow core. In this case, the amount of recycled gas raining down from the innermost region may depend on other unknown factors. For instance, if secular processes act promptly, compared to the time scales of stellar gas recycle ( $0.1-1$ Gyr $)$, to be able to substantially fill the central region with stars initially formed in outer regions, the SMBH may follow the track we described. If, on ther other hand, secular processes evolve on longer time scales, the recycled stellar gas would predominantly land in outer regions that do not efficiently accrete to the SMBH, which would in turn not grow substantially. It would seem likely that there may be two trends for pseudo-bulges: (1) there will be large variations in $\mathrm{M}_{\mathrm{BH}}-\mathrm{M}_{\mathrm{BG}}$ relation and (2) $\mathrm{SMBH}$ masses may lie below that of the $\mathrm{M}_{\mathrm{BH}}-\mathrm{M}_{\mathrm{BG}}$ relation derived from inactive classic elliptical galaxies/bulges, both consistent with independent considerations in the context of hierarchical structure formation model (e.g., Shankar et al. 2012). Observations, while very challenging, may have already provided some hints of both (Greene et al. 2008).

Moreover, we do not expect any discernible correlation between the SMBH and galaxy disk or dark matter halo, simply because the stars in disks do not affect SMBH growth and the overall dark matter halo, while indirectly affect the escape velocity that enters Equation 18), does not control the amount of gas that feeds the SMBH. This prediction is consistent with observations (e.g., Kormendy \& Bender 2011). In addition, some stellar population in the outskirts (either on a disk or just at large radii of an elliptical galaxy) of AGN hosts may be unrelated to the preceding starburst and could be substantially different from bulge stars (e.g., Nolan et al. 2001). 


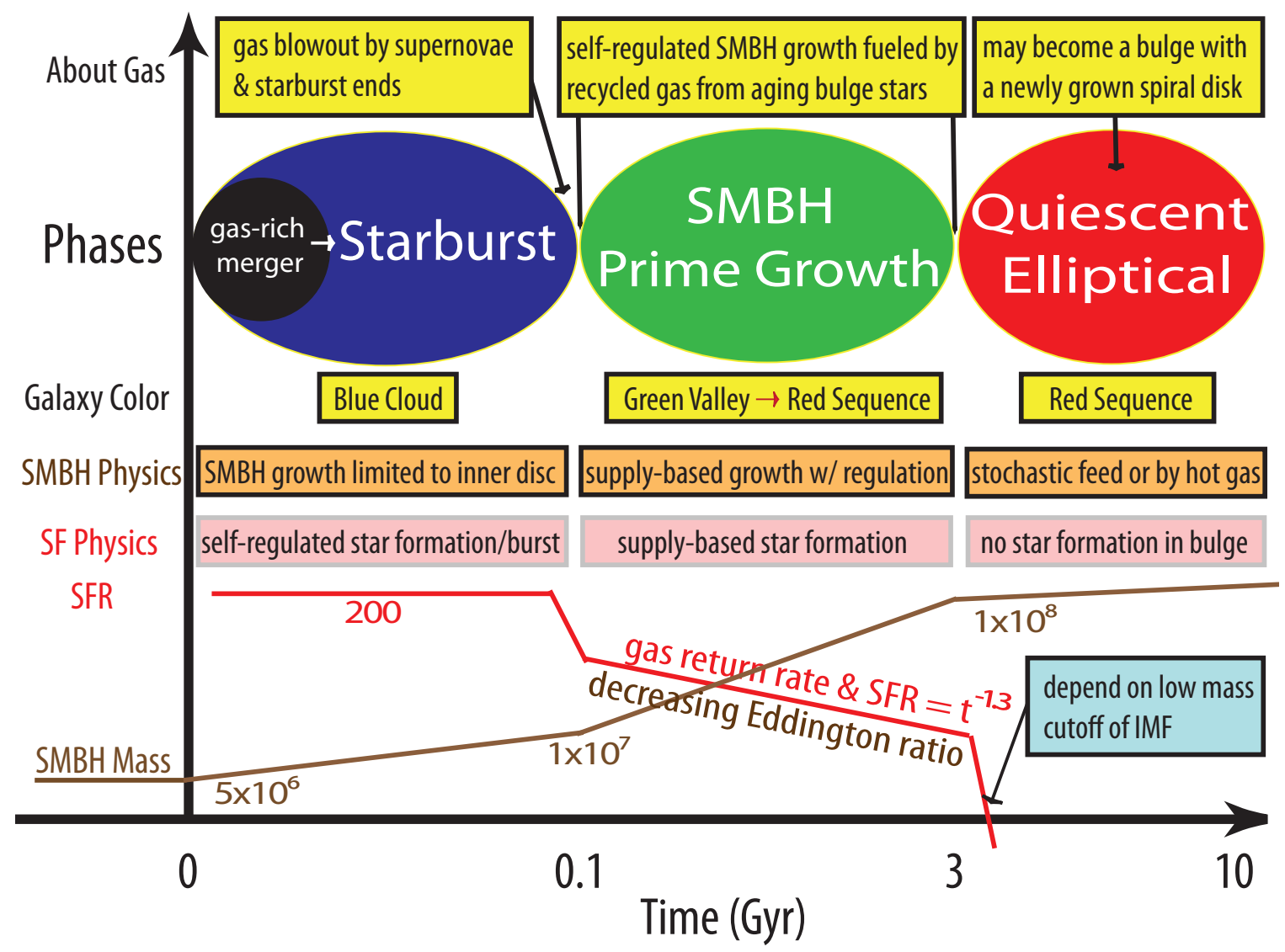

Fig. 2. - shows the entire evolutionary process for an example merger of two gas-rich spirals of mass $\sim 10^{12} \mathrm{M}_{\odot}$ each that eventually produces a powerlaw elliptical galaxy of velocity dispersion of $200 \mathrm{~km} / \mathrm{s}$. This scenario is not limitd to merger events but encompasses any significant event triggering a starburst. Note that the time boundaries between difference consecutive phases are approximate and uncertain to within a factor of a few. The numbers in brown indicate the BH masses and the numbers in red indicate SFR. These numbers are very approximate and given mainly for illustration purpose. Clearly, given the complexity, one should expect large variations from case to case. 

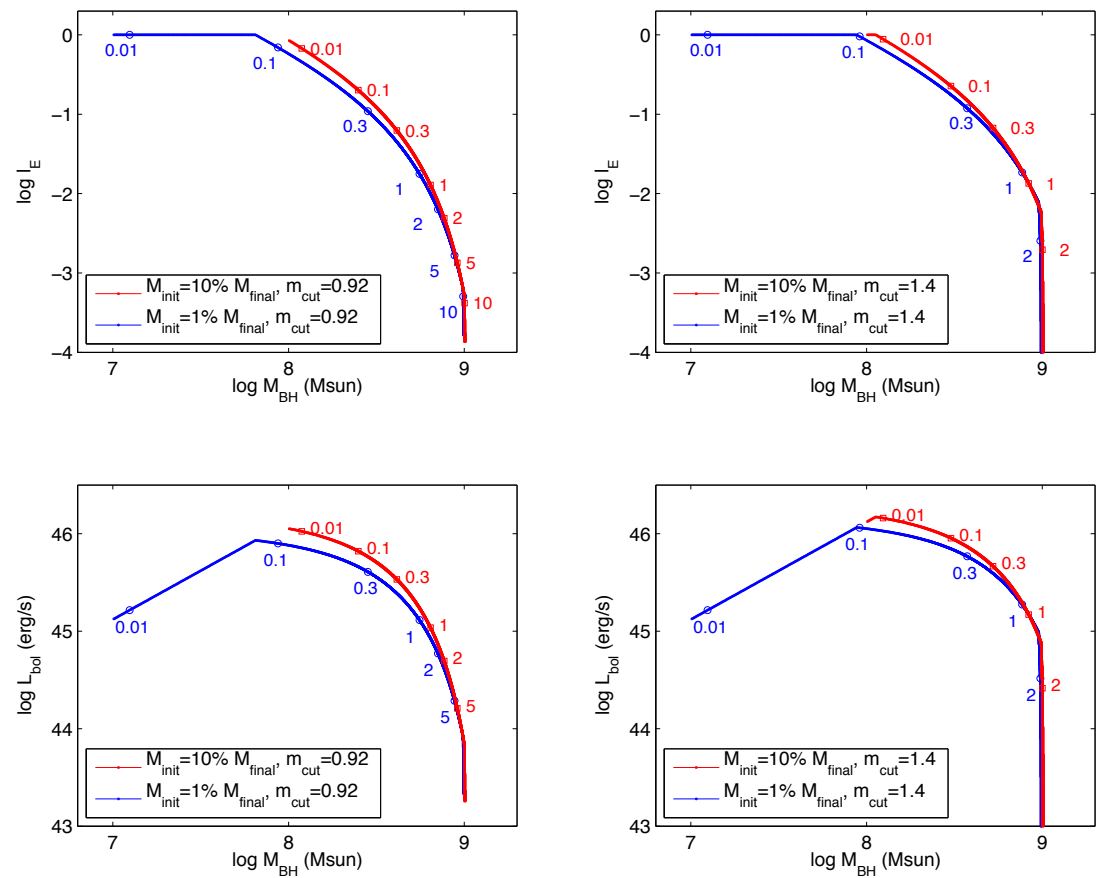

Fig. 3.- Top left panel: evolutionary growth tracks in the SMBH mass- Eddington ratio plane of an example SMBH of final mass $10^{9} \mathrm{M}_{\odot}$ with two cases of seed black mass of $10^{7}$ and $10^{8} \mathrm{M}_{\odot}$, respectively. A low mass cutoff for the IMF of $0.92 \mathrm{M}_{\odot}$ that has a turnoff lifetime of 10 Gyr is assumed. We assume that the SMBH accretion rate is proportional to the recycle gas return rate of the form $\propto t^{-1.3}$ Ciotti et al. (1991) capped at the Eddington rate with a radiative efficiency of $\epsilon=0.1$, starting 200Myrs after the end of the starburst. Also indicated along each track are the times in Gyrs elapsed since the start of the accretion. Top right panel: the case for a low mass cutoff for the IMF of $1.4 \mathrm{M}_{\odot}$ that has a turnoff lifetime of 2 Gyr. Bottom panels: tracks for the cases in top panels but in the SMBH mass-luminosity plane. 


\section{Conclusions}

We have presented an alternative physical model that has the following characteristics for the coevolution of galaxy and SMBH. From the onset of a starburst to becoming a quiescent bulge (in the absence of any subsequent significant burst event) there are three distinct periods:

(1) "Starburst Period": some significant event induces a starburst that probably lasts about $10^{7}-10^{8}$ yrs. The SMBH grows modestly during this period to possibly attain a mass that is up to order ten percent of its final mass. The feedback energy/momentum from the starburst, i.e., supernovae, drives the last patch of gas away and shuts down star formation.

(2) "SMBH Prime Period": several hundred million years after the end of the starburst, aging low-to-intermediate mass stars, now in their post-main-sequence phases, start to return a substantial fraction of their stellar mass to the ISM. Because the rate of gas return from stars diminishes with time, the Eddington ratio of the SMBH decreases with time roughly as $t^{-1.3}$. The SMBH growth is synchronous with star formation from recycled gas during this period. The accompanying star formation rate may also be substantial. The duration of this phase depends sensitively on the lower cutoff mass of the initial mass function (IMF).

(3) "Quiescent Bulge": on order of gigayear after the end of the starburst the elliptical galaxy is now truly red and dead - gas return rate is now negligible so both accretion to the central SMBH and residual star formation have ceased. It is possible that a disk may grow around the bulge later. The feeding of the central SMBH in the bulge of spiral galaxy during this period is not by overhead material from aging stars, rather by occasional objects that happen to be on some plunging orbits to be disrupted by the SMBH and form a short-lived accretion disc. Candidate objects may include molecular clouds or tidally disrupted stars.

In this model, the end of starburst precedes the onset of prime SMBH growth by order of 100Myr. Starburst is responsible for shutting down its own activities; AGN has little to do with it. AGN does provide self-regulation during its prime growth post-starburst period. An important feature of this model is that it is physically based and no significant fine tuning is required. The physical reason why the SMBH does not grow substantially in the starburst phase, although over-supplied with gas, is that only a very small central disc is gravitationally stable for gas accretion onto the $\mathrm{SMBH}$, while all other regions are unstable and more conducive to star formation. The condition is just the opposite during the post-starburst phase where recycled gas dropout from aging stars returns slowly and can be more effectively accreted, so effective that self-regulation comes to play, energetically feasibly provided by the radiation pressure.

Many comparisons between this physical model and extant observations are made and the model appears to be in very agreement with them, including the $\mathrm{M}_{\mathrm{BH}}-\mathrm{M}_{\mathrm{BG}}$ relation. 
This model predicts that the distribution of the Eddington ratio of AGNs in star-forming galaxies is lognormal, whereas that of AGNs in early type galaxies is a powerlaw, consistent with observations (Kauffmann \& Heckman 2009). We predict that early-type galaxy hosts of high Eddingtion rate AGNs are expected to be light-blue to green in optical color, gradually evolving to the red sequences with decreasing AGN luminosity.

I thank Jerry Ostriker for useful comments and discussion. I would also like to thank Greg Novak, Kevin Schawinski and Charles Steinhardt for useful discussion. I thank an anonymous referee for critical and constructive reports. This work is supported in part by grants NNX08AH31G and NNX11AI23G.

\section{REFERENCES}

Adelberger, K. L., Steidel, C. C., Pettini, M., Shapley, A. E., Reddy, N. A., \& Erb, D. K. 2005, ApJ, 619, 697

Aird, J., Coil, A. L., Moustakas, J., Blanton, M. R., Burles, S. M., Cool, R. J., Eisenstein, D. J., Smith, M. S. M., Wong, K. C., \& Zhu, G. 2012, ApJ, 746, 90

Alexander, D. M., Bauer, F. E., Chapman, S. C., Smail, I., Blain, A. W., Brandt, W. N., \& Ivison, R. J. 2005a, ApJ, 632, 736

Alexander, D. M., Brandt, W. N., Smail, I., Swinbank, A. M., Bauer, F. E., Blain, A. W., Chapman, S. C., Coppin, K. E. K., Ivison, R. J., \& Menéndez-Delmestre, K. 2008, AJ, 135, 1968

Alexander, D. M., Smail, I., Bauer, F. E., Chapman, S. C., Blain, A. W., Brandt, W. N., \& Ivison, R. J. 2005b, Nature, 434, 738

Alexander, D. M., Swinbank, A. M., Smail, I., McDermid, R., \& Nesvadba, N. P. H. 2010, MNRAS, 402, 2211

Allen, S. W., Dunn, R. J. H., Fabian, A. C., Taylor, G. B., \& Reynolds, C. S. 2006, MNRAS, 372,21

Armitage, P. J. 1998, ApJ, 501, L189+

Bahcall, J. N., Kirhakos, S., Saxe, D. H., \& Schneider, D. P. 1997, ApJ, 479, 642

Balbus, S. A., \& Hawley, J. F. 1991, ApJ, 376, 214

Balmaverde, B., \& Capetti, A. 2006, A\&A, 447, 97 
Barger, A. J., Cowie, L. L., Mushotzky, R. F., Yang, Y., et al. 2005, AJ, 129, 578

Bender, R., Kormendy, J., Bower, G., Green, R., Thomas, J., Danks, A. C., Gull, T., Hutchings, J. B., Joseph, C. L., Kaiser, M. E., Lauer, T. R., Nelson, C. H., Richstone, D., Weistrop, D., \& Woodgate, B. 2005, ApJ, 631, 280

Bennert, N., Canalizo, G., Jungwiert, B., Stockton, A., Schweizer, F., Peng, C. Y., \& Lacy, M. 2008, ApJ, 677, 846

Best, P. N., Kauffmann, G., Heckman, T. M., Brinchmann, J., Charlot, S., Ivezić, Ž., \& White, S. D. M. 2005, MNRAS, 362, 25

Blanton, M. R., Hogg, D. W., Bahcall, N. A., Baldry, I. K., Brinkmann, J., Csabai, I., Eisenstein, D., Fukugita, M., Gunn, J. E., Ivezić, Ž., Lamb, D. Q., Lupton, R. H., Loveday, J., Munn, J. A., Nichol, R. C., Okamura, S., Schlegel, D. J., Shimasaku, K., Strauss, M. A., Vogeley, M. S., \& Weinberg, D. H. 2003, ApJ, 594, 186

Boyle, B. J., Shanks, T., \& Peterson, B. A. 1988, MNRAS, 235, 935

Brandenburg, A., Nordlund, A., Stein, R. F., \& Torkelsson, U. 1995, ApJ, 446, 741

Brown, M. J. I., Zheng, Z., White, M., Dey, A., Jannuzi, B. T., Benson, A. J., Brand, K., Brodwin, M., \& Croton, D. J. 2008, ApJ, 682, 937

Canalizo, G., \& Stockton, A. 2001, ApJ, 555, 719

Chapman, S. C., Blain, A. W., Smail, I., \& Ivison, R. J. 2005, ApJ, 622, 772

Choi, E., \& Ostriker, J. P. 2011, ApJ

Ciotti, L., D’Ercole, A., Pellegrini, S., \& Renzini, A. 1991, ApJ, 376, 380

Ciotti, L., \& Ostriker, J. P. 2007, ApJ, 665, 1038

Cirasuolo, M., Shankar, F., Granato, G. L., De Zotti, G., \& Danese, L. 2005, ApJ, 629, 816

Cisternas, M., Jahnke, K., Inskip, K. J., Kartaltepe, J., Koekemoer, A. M., Lisker, T., Robaina, A. R., Scodeggio, M., Sheth, K., Trump, J. R., Andrae, R., Miyaji, T., Lusso, E., Brusa, M., Capak, P., Cappelluti, N., Civano, F., Ilbert, O., Impey, C. D., Leauthaud, A., Lilly, S. J., Salvato, M., Scoville, N. Z., \& Taniguchi, Y. 2011, ApJ, 726,57

Cowie, L. L., Songaila, A., Hu, E. M., \& Cohen, J. G. 1996, AJ, 112, 839

Crenshaw, D. M., Kraemer, S. B., \& George, I. M. 2003, ARA\&A, 41, 117 
Croton, D. J., Springel, V., White, S. D. M., De Lucia, G., Frenk, C. S., Gao, L., Jenkins, A., Kauffmann, G., Navarro, J. F., \& Yoshida, N. 2006, MNRAS, 365, 11

DeBuhr, J., Quataert, E., \& Ma, C. 2010, ArXiv e-prints

Di Matteo, T., Springel, V., \& Hernquist, L. 2005, Nature, 433, 604

Downes, D., \& Solomon, P. M. 1998, ApJ, 507, 615

Dunlop, J. S., McLure, R. J., Kukula, M. J., Baum, S. A., O’Dea, C. P., \& Hughes, D. H. 2003, MNRAS, 340, 1095

Dunn, J. P., Bautista, M., Arav, N., Moe, M., Korista, K., Costantini, E., Benn, C., Ellison, S., \& Edmonds, D. 2010, ApJ, 709, 611

Elvis, M., Wilkes, B. J., McDowell, J. C., Green, R. F., Bechtold, J., Willner, S. P., Oey, M. S., Polomski, E., \& Cutri, R. 1994, ApJS, 95, 1

Emonts, B. H. C., Morganti, R., Tadhunter, C. N., Holt, J., Oosterloo, T. A., van der Hulst, J. M., \& Wills, K. A. 2006, A\&A, 454, 125

Evans, A. S., Sanders, D. B., Surace, J. A., \& Mazzarella, J. M. 1999, ApJ, 511, 730

Faber, S. M., Tremaine, S., Ajhar, E. A., Byun, Y., Dressler, A., Gebhardt, K., Grillmair, C., Kormendy, J., Lauer, T. R., \& Richstone, D. 1997, AJ, 114, 1771

Fan, X., Strauss, M. A., Richards, G. T., Hennawi, J. F., Becker, R. H., White, R. L., Diamond-Stanic, A. M., Donley, J. L., Jiang, L., Kim, J. S., Vestergaard, M., Young, J. E., Gunn, J. E., Lupton, R. H., Knapp, G. R., Schneider, D. P., Brandt, W. N., Bahcall, N. A., Barentine, J. C., Brinkmann, J., Brewington, H. J., Fukugita, M., Harvanek, M., Kleinman, S. J., Krzesinski, J., Long, D., Neilsen, Jr., E. H., Nitta, A., Snedden, S. A., \& Voges, W. 2006, AJ, 131, 1203

Ferrarese, L., \& Merritt, D. 2000, ApJ, 539, L9

Fleming, T., \& Stone, J. M. 2003, ApJ, 585, 908

Frank, J., King, A., \& Raine, D. 1992, Accretion power in astrophysics., ed. Frank, J., King, A., \& Raine, D.

Fromang, S., \& Papaloizou, J. 2007, A\&A, 476, 1113

Gammie, C. F. 2001, ApJ, 553, 174

Gao, Y., \& Solomon, P. M. 2004, ApJ, 606, 271 
Genzel, R., Lutz, D., Sturm, E., Egami, E., Kunze, D., Moorwood, A. F. M., Rigopoulou, D., Spoon, H. W. W., Sternberg, A., Tacconi-Garman, L. E., Tacconi, L., \& Thatte, N. 1998, ApJ, 498, 579

Georgakakis, A., Nandra, K., Yan, R., Willner, S. P., Lotz, J. M., Pierce, C. M., Cooper, M. C., Laird, E. S., Koo, D. C., Barmby, P., Newman, J. A., Primack, J. R., \& Coil, A. L. 2008, MNRAS, 385, 2049

Goodman, J. 2003, MNRAS, 339, 937

Granato, G. L., De Zotti, G., Silva, L., Bressan, A., \& Danese, L. 2004, ApJ, 600, 580

Granato, G. L., Silva, L., Lapi, A., Shankar, F., De Zotti, G., \& Danese, L. 2006, MNRAS, 368, L72

Greene, J. E., Ho, L. C., \& Barth, A. J. 2008, ApJ, 688, 159

Greene, J. E., Zakamska, N. L., Ho, L. C., \& Barth, A. J. 2011, ArXiv e-prints

Grogin, N. A., Conselice, C. J., Chatzichristou, E., Alexander, D. M., Bauer, F. E., Hornschemeier, A. E., Jogee, S., Koekemoer, A. M., Laidler, V. G., Livio, M., Lucas, R. A., Paolillo, M., Ravindranath, S., Schreier, E. J., Simmons, B. D., \& Urry, C. M. 2005, ApJ, 627, L97

Hamann, F., \& Ferland, G. 1993, ApJ, 418, 11

-. 1999, ARA\&A, 37, 487

Hasinger, G., Miyaji, T., \& Schmidt, M. 2005, A\&A, 441, 417

Hawley, J. F., Gammie, C. F., \& Balbus, S. A. 1995, ApJ, 440, 742

Heckman, T. M. 2001, in Astronomical Society of the Pacific Conference Series, Vol. 240, Gas and Galaxy Evolution, ed. J. E. Hibbard, M. Rupen, \& J. H. van Gorkom, 345

Hennawi, J. F., Myers, A. D., Shen, Y., Strauss, M. A., Djorgovski, S. G., Fan, X., Glikman, E., Mahabal, A., Martin, C. L., Richards, G. T., Schneider, D. P., \& Shankar, F. 2010, ApJ, 719, 1672

Hickox, R. C., Jones, C., Forman, W. R., Murray, S. S., Kochanek, C. S., Eisenstein, D., Jannuzi, B. T., Dey, A., Brown, M. J. I., Stern, D., Eisenhardt, P. R., Gorjian, V., Brodwin, M., Narayan, R., Cool, R. J., Kenter, A., Caldwell, N., \& Anderson, M. E. 2009, ApJ, 696, 891

Hopkins, P. F., Hernquist, L., Cox, T. J., Di Matteo, T., Robertson, B., \& Springel, V. 2006, ApJS, 163, 1 
Hopkins, P. F., Lauer, T. R., Cox, T. J., Hernquist, L., \& Kormendy, J. 2009, ApJS, 181, 486

Ivison, R. J., Greve, T. R., Serjeant, S., Bertoldi, F., Egami, E., Mortier, A. M. J., AlonsoHerrero, A., Barmby, P., Bei, L., Dole, H., Engelbracht, C. W., Fazio, G. G., Frayer, D. T., Gordon, K. D., Hines, D. C., Huang, J., Le Floc'h, E., Misselt, K. A., Miyazaki, S., Morrison, J. E., Papovich, C., Pérez-González, P. G., Rieke, M. J., Rieke, G. H., Rigby, J., Rigopoulou, D., Smail, I., Wilson, G., \& Willner, S. P. 2004, ApJS, 154, 124

Ivison, R. J., Smail, I., Barger, A. J., Kneib, J., Blain, A. W., Owen, F. N., Kerr, T. H., \& Cowie, L. L. 2000, MNRAS, 315, 209

Jaffe, W., Meisenheimer, K., Röttgering, H. J. A., Leinert, C., Richichi, A., Chesneau, O., Fraix-Burnet, D., Glazenborg-Kluttig, A., Granato, G., Graser, U., Heijligers, B., Köhler, R., Malbet, F., Miley, G. K., Paresce, F., Pel, J., Perrin, G., Przygodda, F., Schoeller, M., Sol, H., Waters, L. B. F. M., Weigelt, G., Woillez, J., \& de Zeeuw, P. T. 2004, Nature, 429, 47

Jahnke, K., Sánchez, S. F., Wisotzki, L., Barden, M., Beckwith, S. V. W., Bell, E. F., Borch, A., Caldwell, J. A. R., Häussler, B., Heymans, C., Jogee, S., McIntosh, D. H., Meisenheimer, K., Peng, C. Y., Rix, H., Somerville, R. S., \& Wolf, C. 2004, ApJ, 614, 568

Johnson, B. M., \& Gammie, C. F. 2003, ApJ, 597, 131

Kauffmann, G., \& Haehnelt, M. 2000, MNRAS, 311, 576

Kauffmann, G., \& Heckman, T. M. 2009, MNRAS, 397, 135

Kauffmann, G., Heckman, T. M., White, S. D. M., Charlot, S., Tremonti, C., Peng, E. W., Seibert, M., Brinkmann, J., Nichol, R. C., SubbaRao, M., \& York, D. 2003, MNRAS, 341,54

Kaviraj, S. 2009, MNRAS, 394, 1167

Kaviraj, S., Schawinski, K., Silk, J., \& Shabala, S. S. 2010, ArXiv e-prints

Kawakatu, N., Anabuki, N., Nagao, T., Umemura, M., \& Nakagawa, T. 2006, ApJ, 637, 104

Kormendy, J., \& Bender, R. 2011, Nature, 469, 377

Kormendy, J., Fisher, D. B., Cornell, M. E., \& Bender, R. 2009, ApJS, 182, 216

Kormendy, J., \& Kennicutt, Jr., R. C. 2004, ARA\&A, 42, 603 
Kurosawa, R., \& Proga, D. 2009, MNRAS, 397, 1791

Lamastra, A., Menci, N., Maiolino, R., Fiore, F., \& Merloni, A. 2010, MNRAS, 405, 29

Lapi, A., Shankar, F., Mao, J., Granato, G. L., Silva, L., De Zotti, G., \& Danese, L. 2006, ApJ, 650, 42

Ledlow, M. J., Owen, F. N., Yun, M. S., \& Hill, J. M. 2001, ApJ, 552, 120

Levin, Y., \& Beloborodov, A. M. 2003, ApJ, 590, L33

Lu, J. R., Ghez, A. M., Hornstein, S. D., Morris, M. R., Becklin, E. E., \& Matthews, K. 2009, ApJ, 690, 1463

Lutz, D., Spoon, H. W. W., Rigopoulou, D., Moorwood, A. F. M., \& Genzel, R. 1998, ApJ, 505, L103

Magorrian, J., Tremaine, S., Richstone, D., Bender, R., Bower, G., Dressler, A., Faber, S. M., Gebhardt, K., Green, R., Grillmair, C., Kormendy, J., \& Lauer, T. 1998, AJ, 115,2285

Marconi, A., \& Hunt, L. K. 2003, ApJ, 589, L21

Marconi, A., Risaliti, G., Gilli, R., Hunt, L. K., Maiolino, R., \& Salvati, M. 2004, MNRAS, 351,169

Martini, P. 2004, Coevolution of Black Holes and Galaxies, 169

Mathews, W. G. 1989, AJ, 97, 42

McKee, C. F., \& Ostriker, E. C. 2007, ARA\&A, 45, 565

Mendez, A. J., Coil, A. L., Lotz, J., Salim, S., Moustakas, J., \& Simard, L. 2011, ArXiv e-prints

Milosavljević, M., \& Merritt, D. 2001, ApJ, 563, 34

Moe, M., Arav, N., Bautista, M. A., \& Korista, K. T. 2009, ApJ, 706, 525

Murray, N., Chiang, J., Grossman, S. A., \& Voit, G. M. 1995, ApJ, 451, 498

Nandra, K., Laird, E. S., \& Steidel, C. C. 2005, MNRAS, 360, L39

Nolan, L. A., Dunlop, J. S., Kukula, M. J., Hughes, D. H., Boroson, T., \& Jimenez, R. 2001, MNRAS, 323, 308

Norman, C., \& Scoville, N. 1988, ApJ, 332, 124 
Novak, G. S., Ostriker, J. P., \& Ciotti, L. 2010, ArXiv e-prints

Paumard, T., Genzel, R., Martins, F., Nayakshin, S., Beloborodov, A. M., Levin, Y., Trippe, S., Eisenhauer, F., Ott, T., Gillessen, S., Abuter, R., Cuadra, J., Alexander, T., \& Sternberg, A. 2006, ApJ, 643, 1011

Pellegrini, S. 2005, MNRAS, 364, 169

Ptak, A., Heckman, T., Levenson, N. A., Weaver, K., \& Strickland, D. 2003, ApJ, 592, 782

Ranalli, P., Comastri, A., \& Setti, G. 2003, A\&A, 399, 39

Rauch, K. P., \& Tremaine, S. 1996, New A, 1, 149

Rice, W. K. M., Armitage, P. J., Bate, M. R., \& Bonnell, I. A. 2003, MNRAS, 339, 1025

Richstone, D., Ajhar, E. A., Bender, R., Bower, G., Dressler, A., Faber, S. M., Filippenko, A. V., Gebhardt, K., Green, R., Ho, L. C., Kormendy, J., Lauer, T. R., Magorrian, J., \& Tremaine, S. 1998, Nature, 395, A14+

Risaliti, G., Maiolino, R., \& Salvati, M. 1999, ApJ, 522, 157

Salim, S., Rich, R. M., Charlot, S., Brinchmann, J., Johnson, B. D., Schiminovich, D., Seibert, M., Mallery, R., Heckman, T. M., Forster, K., Friedman, P. G., Martin, D. C., Morrissey, P., Neff, S. G., Small, T., Wyder, T. K., Bianchi, L., Donas, J., Lee, Y., Madore, B. F., Milliard, B., Szalay, A. S., Welsh, B. Y., \& Yi, S. K. 2007, ApJS, 173, 267

Sánchez, S. F., Jahnke, K., Wisotzki, L., McIntosh, D. H., Bell, E. F., Barden, M., Beckwith, S. V. W., Borch, A., Caldwell, J. A. R., Häussler, B., Jogee, S., Meisenheimer, K., Peng, C. Y., Rix, H., Somerville, R. S., \& Wolf, C. 2004, ApJ, 614, 586

Sanders, D. B., Soifer, B. T., Elias, J. H., Madore, B. F., Matthews, K., Neugebauer, G., \& Scoville, N. Z. 1988, ApJ, 325, 74

Sazonov, S. Y., Ostriker, J. P., \& Sunyaev, R. A. 2004, MNRAS, 347, 144

Schawinski, K., Urry, C. M., Virani, S., Coppi, P., Bamford, S. P., Treister, E., Lintott, C. J., Sarzi, M., Keel, W. C., Kaviraj, S., Cardamone, C. N., Masters, K. L., Ross, N. P., Andreescu, D., Murray, P., Nichol, R. C., Raddick, M. J., Slosar, A., Szalay, A. S., Thomas, D., \& Vandenberg, J. 2010, ApJ, 711, 284

Schawinski, K., Virani, S., Simmons, B., Urry, C. M., Treister, E., Kaviraj, S., \& Kushkuley, B. 2009, ApJ, 692, L19 
Schweitzer, M., Lutz, D., Sturm, E., Contursi, A., Tacconi, L. J., Lehnert, M. D., Dasyra, K. M., Genzel, R., Veilleux, S., Rupke, D., Kim, D., Baker, A. J., Netzer, H., Sternberg, A., Mazzarella, J., \& Lord, S. 2006, ApJ, 649, 79

Shakura, N. I., \& Sunyaev, R. A. 1973, A\&A, 24, 337

Shankar, F., Marulli, F., Mathur, S., Bernardi, M., \& Bournaud, F. 2012, A\&A, 540, A23

Shi, Y., Rieke, G. H., Ogle, P., Jiang, L., \& Diamond-Stanic, A. M. 2009, ApJ, 703, 1107

Silk, J., \& Nusser, A. 2010, ApJ, 725, 556

Silk, J., \& Rees, M. J. 1998, A\&A, 331, L1

Silverman, J. D., Lamareille, F., Maier, C., Lilly, S. J., Mainieri, V., Brusa, M., Cappelluti, N., Hasinger, G., Zamorani, G., Scodeggio, M., Bolzonella, M., Contini, T., Carollo, C. M., Jahnke, K., Kneib, J., Le Fèvre, O., Merloni, A., Bardelli, S., Bongiorno, A., Brunner, H., Caputi, K., Civano, F., Comastri, A., Coppa, G., Cucciati, O., de la Torre, S., de Ravel, L., Elvis, M., Finoguenov, A., Fiore, F., Franzetti, P., Garilli, B., Gilli, R., Iovino, A., Kampczyk, P., Knobel, C., Kovač, K., Le Borgne, J., Le Brun, V., Mignoli, M., Pello, R., Peng, Y., Perez Montero, E., Ricciardelli, E., Tanaka, M., Tasca, L., Tresse, L., Vergani, D., Vignali, C., Zucca, E., Bottini, D., Cappi, A., Cassata, P., Fumana, M., Griffiths, R., Kartaltepe, J., Koekemoer, A., Marinoni, C., McCracken, H. J., Memeo, P., Meneux, B., Oesch, P., Porciani, C., \& Salvato, M. 2009, ApJ, 696, 396

Silverman, J. D., Mainieri, V., Lehmer, B. D., Alexander, D. M., Bauer, F. E., Bergeron, J., Brandt, W. N., Gilli, R., Hasinger, G., Schneider, D. P., Tozzi, P., Vignali, C., Koekemoer, A. M., Miyaji, T., Popesso, P., Rosati, P., \& Szokoly, G. 2008, ApJ, 675, 1025

Simões Lopes, R. D., Storchi-Bergmann, T., de Fátima Saraiva, M., \& Martini, P. 2007, ApJ, 655, 718

Smith, K. L., Shields, G. A., Bonning, E. W., McMullen, C. C., Rosario, D. J., \& Salviander, S. 2010, ApJ, 716, 866

Soker, N., \& Meiron, Y. 2010, MNRAS, 1833

Somerville, R. S., Hopkins, P. F., Cox, T. J., Robertson, B. E., \& Hernquist, L. 2008, MNRAS, 391, 481

Steinhardt, C. L., \& Elvis, M. 2010, MNRAS, 402, 2637

Stone, J. M., Hawley, J. F., Gammie, C. F., \& Balbus, S. A. 1996, ApJ, 463, 656 
Tremaine, S., Gebhardt, K., Bender, R., Bower, G., Dressler, A., Faber, S. M., Filippenko, A. V., Green, R., et al. 2002, ApJ, 574, 740

Treu, T., Ellis, R. S., Liao, T. X., \& van Dokkum, P. G. 2005, ApJ, 622, L5

van der Tak, F. F. S., \& van Dishoeck, E. F. 2000, A\&A, 358, L79

Veilleux, S., Rupke, D. S. N., Kim, D., Genzel, R., Sturm, E., Lutz, D., Contursi, A., Schweitzer, M., Tacconi, L. J., Netzer, H., Sternberg, A., Mihos, J. C., Baker, A. J., Mazzarella, J. M., Lord, S., Sanders, D. B., Stockton, A., Joseph, R. D., \& Barnes, J. E. 2009, ApJS, 182, 628

Véron-Cetty, M. P., \& Véron, P. 2001, A\&A, 375, 791

Weiner, B. J., Coil, A. L., Prochaska, J. X., Newman, J. A., Cooper, M. C., Bundy, K., Conselice, C. J., Dutton, A. A., Faber, S. M., Koo, D. C., Lotz, J. M., Rieke, G. H., \& Rubin, K. H. R. 2009, ApJ, 692, 187

Woo, J., \& Urry, C. M. 2002, ApJ, 579, 530

Yu, Q., \& Tremaine, S. 2002, MNRAS, 335, 965

This preprint was prepared with the AAS IATEX macros v5.2. 